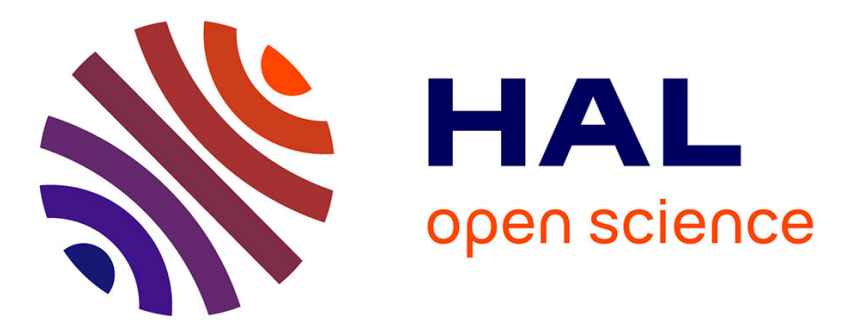

\title{
Periodic homogenization for fully coupled thermomechanical modeling of dissipative generalized standard materials
}

Georges Chatzigeorgiou, Nicolas Charalambakis, Yves Chemisky, Fodil

Meraghni

\section{To cite this version:}

Georges Chatzigeorgiou, Nicolas Charalambakis, Yves Chemisky, Fodil Meraghni. Periodic homogenization for fully coupled thermomechanical modeling of dissipative generalized standard materials. International Journal of Plasticity, 2016, 81, pp.18-39. 10.1016/j.ijplas.2016.01.013 . hal-01515204

\section{HAL Id: hal-01515204 \\ https://hal.univ-lorraine.fr/hal-01515204}

Submitted on 31 Mar 2020

HAL is a multi-disciplinary open access archive for the deposit and dissemination of scientific research documents, whether they are published or not. The documents may come from teaching and research institutions in France or abroad, or from public or private research centers.
L'archive ouverte pluridisciplinaire HAL, est destinée au dépôt et à la diffusion de documents scientifiques de niveau recherche, publiés ou non, émanant des établissements d'enseignement et de recherche français ou étrangers, des laboratoires publics ou privés. 
- Homogenization framework accounting for equilibrium and thermodynamics laws.

- Micro/macro energy potentials and dissipation through asymptotic expansion method.

- Incremental, linearized formulation to obtain macroscale tangent moduli.

- Framework capabilities are illustrated with examples on multilayered composites. 


\title{
Periodic homogenization for fully coupled thermomechanical modeling of dissipative generalized standard materials
}

\author{
George Chatzigeorgiou $^{\mathrm{a}, *}$, Nicolas Charalambakis ${ }^{\mathrm{b}}$, Yves Chemisky $^{\mathrm{a}}$, Fodil Meraghni ${ }^{\mathrm{a}}$ \\ ${ }^{a}$ LEM3-UMR 7239 CNRS, Arts et Métiers ParisTech Metz-Lorraine, 4 Rue Augustin Fresnel 57078 Metz, France \\ ${ }^{b}$ Department of Civil Engineering, Aristotle University of Thessaloniki, GR 54124, Thessaloniki, Greece
}

\begin{abstract}
The current work deals with periodic thermomechanical composite media, in which the material constituents are considered to obey the generalized standard materials laws. The aim is to provide a proper homogenization framework that takes into account both the equilibrium and the thermodynamics laws in microscale and macroscale levels. The study is based on the asymptotic expansion homogenization technique, which permits to deduce useful results about the general structure of microscale and macroscale energy potentials and constitutive laws. The paper also proposes an incremental, linearized formulation that allows to identify suitable thermomechanical tangent moduli for the macroscale problem. The capabilities of this framework are illustrated with numerical examples on multilayered composites.
\end{abstract}

Keywords: A. thermomechanical processes; B. elastic-viscoplastic material; B. layered material; C. asymptotic analysis; periodic homogenization.

\section{Introduction}

The recent advances of the materials science and the increased needs of many engineering applications has led to the tremendous growth of composite materials. The automotive and aerospace industry require smart composites that are able to be utilized in complicated structures with high demands in strength, multifunctionality and durability, and at the same time to have long lifetime during repeated loading cycles.

To match those high requirements, composite materials are often utilized in regimes where dissipative phenomena occur, i.e viscoelasticity, viscoplasticity, plasticity or phase transformation. Such mechanisms may be accompanied by a significant temperature change, which influences in return the material behavior. To account for such thermomechanical coupling is especially important in the case of polymers being utilized in temperature ranges close to the glass transition zones, or in shape memory alloys, and is a key feature to accurately predict fatigue in composite materials (Benaarbia et al., 2015).

Email addresses: georges.chatzigeorgiou@ensam.eu (George Chatzigeorgiou), charalam@civil.auth.gr (Nicolas Charalambakis), yves.chemisky@ensam.eu (Yves Chemisky), fodil.meraghni@ensam.eu (Fodil Meraghni) 
Such strong couplings have been already taken into account in the constitutive equations, e.g. in the generalized standard material definition (Germain, 1973; Halphen and Nguyen, 1975; Germain, 1982; Germain et al., 1983). However, in the case of composite materials, very few works have been dedicated to the study of the fully coupled thermomechanical behavior of composites. This topic is nowadays of a great importance since, for instance, fatigue of composites is a complicated mechanism that depends not only on the stress state, but also on the energy dissipation that occurs during inelastic mechanisms. Thus, an appropriate study of the fatigue of a structure requires to consider the energy exchanges during thermomechanical loading cycles.

The scope of the current manuscript is to identify, through the asymptotic expansion method, a proper, fully coupled, thermomechanical homogenization framework for the study of periodic composites consisting of generalized standard materials. The response of generalized standard materials is properly described by a set of suitable state and internal variables. Thermodynamic forces related with these variables can be obtained through the definition of a free energy potential. Additionally, the introduction of a dissipation potential allows to define evolution equations for the internal variables from the dissipation inequality. In composites, the dissipation inequality should be satisfied in both micro and macro levels in order to lead to accurate constitutive evolution equations for the internal variables.

Modeling the mechanical behavior of heterogeneous, nonlinear, dissipative composite materials has been the subject of many researchers. Several multiscale techniques have been proposed in order to identify the overall behavior of viscoelastic, elastoplastic, viscoplastic, or damaged composite materials (Suquet, 1987; Ponte-Castañeda, 1991; Terada and Kikuchi, 2001; Desrumaux et al., 2001; Meraghni et al., 2002; Yu and Fish, 2002; Aboudi et al., 2003; Aboudi, 2004; Chaboche et al., 2005; Love and Batra, 2006; Asada and Ohno, 2007; Jendli et al., 2009; Mercier and Molinari, 2009; Khatam and Pindera, 2010; Cavalcante et al., 2009; Kruch and Chaboche, 2011; Brenner and Suquet, 2013; Tu and Pindera, 2014; Cavalcante and Pindera, 2016). Review papers discussing the various multiscale approaches have been written by Pindera et al. (2009); Charalambakis (2010); Mercier et al. (2012).

In the homogenization theories for generalized standard materials with nonlinear dissipative mechanisms there is no general and unique way to express the two potentials (Helmholtz or Gibbs and the dissipation potential) for the macroscopic composite behavior. Many proposed methodologies in the literature overcome this difficulty by assuming specific form of the macroscopic response and then obtain the correlation with the microscopic variables (Hutchinson, 1976; Sun and Ju, 2004; Suquet, 2012). Moreover, these works focus exclusively on the mechanical behavior, ignoring the effects of the mechanical work in the energy balance. Fully coupled thermomechanical study for composites under large deformation processes with arbitrary choice of constituents constitutive laws has been performed recently by Sengupta et al. (2012). This work presents original and interesting results, but it is based on certain assumptions about the form of the conservation laws in both scales and the correlation between microscopic and macroscopic variables. Also, no discussion about the thermodynamic micro- and macro-energy potentials is provided.

The asymptotic expansion homogenization method has been developed for periodic media and its advantage is that it does not require initial assumption about the form of the microscopic and macroscopic equations that describe the problem under investigation. It starts with a general form of the system of equations, and through appropriate 
asymptotic expansions on the field variables (displacement, temperature e.t.c.) identifies rigorously how this system is represented in microscale and macroscale (Bensoussan et al., 1978; Sanchez-Palencia, 1978). Nowadays this method is considered classical and fairly straight forward regarding the case of thermoelastic media. In the case of inelastic mechanisms of plasticity type, it has been implemented successfully (see for instance Fish et al., 1997 for composites with plasticity type strains and Herzog and Jacquet, 2007 for shape memory alloy composites), but no discussion about the influence of mechanical dissipation on the temperature of the composite appears in these works. Only a limited number of studies have considered fully coupled thermomechanical processes by combining the conservations of linear momentum and energy (Ene, 1983; Yu and Fish, 2002; Temizer, 2012). Yu and Fish (2002), using the asymptotic expansion homogenization framework, have performed a fully coupled thermomechanical analysis for viscoelastic periodic composites. Their analysis though is based on specific type of constitutive law and the proposed methodology cannot be applied in the same way for other type of inelastic materials.

The novelty and originality of the present work is that it presents a fully coupled thermomechanical homogenization framework under small deformation processes, without considering apriori any specific type of constitutive law, allowing different types of inelastic material behaviors for the various constituents of the composite. The developed methodology identifies initially the micro and macro conservation laws (equilibrium, thermodynamics), and investigates afterwards how a general energy potential (for instance Helmholtz or Gibbs), or more accurately its rate, is properly formulated in both scales. While the tool utilized in this analysis (the asymptotic expansion method) is considered rather classical, to the best of the authors knowledge such type of general investigation has never been performed in the literature. The outcome of this study is that it proves in a systematic and consistent manner which variables and equations in the fully coupled thermomechanical problem can be rigorously identified in the macroscopic scale and which variables require either additional assumptions or numerical treatment (as it is performed here with the linearized incremental formulation).

This paper attempts a systematic study of the homogenization of periodic composites under fully coupled thermomechanical loading conditions. The asymptotic expansion homogenization method is employed in section 2 in order to identify in both micro and macroscale a) the form of the conservation laws (equilibrium, first and second thermodynamic laws) and b) the energy potentials of generalized standard materials, the mechanical dissipation and the connection between the various thermomechanical fields. Section 3 presents a computational scheme based on a linearized incremental form, which allows to obtain numerically macroscopic thermomechanical tangent moduli. A general discussion about the homogenization approach results is provided in section 4. Section 5 presents some illustrative examples on multilayered composites using the proposed framework. The paper is also accompanied by a conclusion section and one appendix. 


\section{Asymptotic expansion homogenization for thermomechanical dissipative materials}

In the current manuscript the indicial notation is adopted and the Einstein summation is considered in double indices. Latin indices take values from 1 to 3 , while greek indices can vary from 1 to an arbitrary integer.

Periodic homogenization considers that a typical composite material or structure is characterized by two scales. The first scale is called microscopic and takes into account the different material constituents and their geometric characteristics inside the microstructure. The second scale is called macroscopic and describes the response of the overall body as if it is a hypothetical homogeneous medium. The scope of homogenization is to identify the connection between the scales in terms of: a) the various fields (mechanical, thermal e.t.c.), and b) the conservation laws and the constitutive relations between the fields.

At the macroscopic scale, the overall body has volume $\bar{V}$ and occupies the space $\overline{\mathcal{D}}$. This space is bounded by a surface $\partial \overline{\mathcal{D}}$ with unit vector $\bar{n}_{i}$. Each macroscopic point is fully identified with the help of a position vector $\bar{x}_{i}$ in $\overline{\mathcal{D}}$. On the other hand, the microscopic level is identified by a unit cell with volume $V$ that occupies the space $\mathcal{D}$. This space is bounded by a surface $\partial \mathcal{D}$ with unit vector $n_{i}$. Each microscopic point in $\mathcal{D}$ is fully described through a position vector $x_{i}$. The two vectors $\bar{x}_{i}$ and $x_{i}$ are connected through the relation $x_{i}=\bar{x}_{i} / \epsilon$, where $\epsilon$ denotes a characteristic length. The periodic homogenization provides accurate results only when the characteristic length is close to zero, i.e. when the microstructure is extremely small compared to the actual size of the composite. Details about the mathematical framework can be found in Bensoussan et al. (1978); Sanchez-Palencia (1978); Allaire (1992); Murat and Tartar (1997).

When considering the composite in a global sense, the general coordinate system $x_{i}^{\epsilon}$ is used, for which the following scale separation rule holds:

$$
\frac{\partial}{\partial x_{i}^{\epsilon}}=\frac{\partial}{\partial \bar{x}_{i}}+\frac{1}{\epsilon} \frac{\partial}{\partial x_{i}}
$$

In the sequel, all the variables that correspond to the general composite response are denoted with a superscript $\epsilon$. Moreover, a bar above a symbol denotes a macroscopic variable that depends exclusively on the vector $\bar{x}_{i}$. Also, the symbol $\langle\{\bullet\}\rangle$ stands for the volume average operator over the unit cell. Finally, a dot above a variable denotes total derivative of the variable with respect to time.

\subsection{Equilibrium and thermodynamic laws}

The equilibrium equation, without inertia and body forces, is written as

$$
\frac{\partial \sigma_{i j}^{\epsilon}}{\partial x_{j}^{\epsilon}}=0,
$$

where $\sigma_{i j}^{\epsilon}$ denotes the stress tensor. In the small strain theory, the kinematics connect the strain tensor $\varepsilon_{i j}^{\epsilon}$ with the displacement vector $u_{i}^{\epsilon}$ through the relation

$$
\varepsilon_{i j}^{\epsilon}=\frac{1}{2}\left[\frac{\partial u_{i}^{\epsilon}}{\partial x_{j}^{\epsilon}}+\frac{\partial u_{j}^{\epsilon}}{\partial x_{i}^{\epsilon}}\right]
$$


In the absence of any source of heat radiation, the equation of energy in the strong form is written as

$$
\frac{\partial q_{i}^{\epsilon}}{\partial x_{i}^{\epsilon}}=\sigma_{i j}^{\epsilon} \dot{\varepsilon}_{i j}^{\epsilon}-\dot{U}^{\epsilon},
$$

where $q_{i}^{\epsilon}$ and $U^{\epsilon}$ denote the heat flux vector and the internal energy per unit volume respectively. The temperature gradient vector $\nabla \theta_{i}^{\epsilon}$ is defined in terms of the absolute temperature $\theta^{\epsilon}$ as

$$
\nabla \theta_{i}^{\epsilon}=\frac{\partial \theta^{\epsilon}}{\partial x_{i}^{\epsilon}}
$$

The second law of thermodynamics in the strong form of Clausius-Duhem inequality is written as

$$
\theta^{\epsilon} \dot{s}^{\epsilon}+\sigma_{i j}^{\epsilon} \dot{\varepsilon}_{i j}^{\epsilon}-\dot{U}^{\epsilon}-\frac{1}{\theta^{\epsilon}} q_{i}^{\epsilon} \nabla \theta_{i}^{\epsilon} \geq 0
$$

with $s^{\epsilon}$ denoting the specific entropy per unit volume. The system of equations (2), (4), (5) and (6) is very general and does not consider any specific form of the internal energy $U^{\epsilon}$ (i.e. does not refer to a specific constitutive law type). The task of this subsection is to identify the correct representation of the system in both micro and macroscale.

The periodicity of the composite microstructure allows to assume the following asymptotic series expansion for the variables of the problem:

$$
\begin{aligned}
u_{i}^{\epsilon}\left(x_{k}^{\epsilon}\right) & =\bar{u}_{i}\left(\bar{x}_{k}\right)+\epsilon u_{i}^{(1)}\left(\bar{x}_{k}, x_{k}\right)+\ldots, \\
\theta^{\epsilon}\left(x_{k}^{\epsilon}\right) & =\bar{\theta}\left(\bar{x}_{k}\right)+\epsilon \theta^{(1)}\left(\bar{x}_{k}, x_{k}\right)+\ldots, \\
\varepsilon_{i j}^{\epsilon}\left(x_{k}^{\epsilon}\right) & =\varepsilon_{i j}^{(0)}\left(\bar{x}_{k}, x_{k}\right)+\epsilon \varepsilon_{i j}^{(1)}\left(\bar{x}_{k}, x_{k}\right)+\ldots, \\
\sigma_{i j}^{\epsilon}\left(x_{k}^{\epsilon}\right) & =\sigma_{i j}^{(0)}\left(\bar{x}_{k}, x_{k}\right)+\epsilon \sigma_{i j}^{(1)}\left(\bar{x}_{k}, x_{k}\right)+\ldots, \\
\nabla \theta_{i}^{\epsilon}\left(x_{k}^{\epsilon}\right) & =\nabla \theta_{i}^{(0)}\left(\bar{x}_{k}, x_{k}\right)+\epsilon \nabla \theta_{i}^{(1)}\left(\bar{x}_{k}, x_{k}\right)+\ldots, \\
q_{i}^{\epsilon}\left(x_{k}^{\epsilon}\right) & =q_{i}^{(0)}\left(\bar{x}_{k}, x_{k}\right)+\epsilon q_{i}^{(1)}\left(\bar{x}_{k}, x_{k}\right)+\ldots, \\
s^{\epsilon}\left(x_{k}^{\epsilon}\right) & =s^{(0)}\left(\bar{x}_{k}, x_{k}\right)+\epsilon s^{(1)}\left(\bar{x}_{k}, x_{k}\right)+\ldots, \\
U^{\epsilon}\left(x_{k}^{\epsilon}\right) & =U^{(0)}\left(\bar{x}_{k}, x_{k}\right)+\epsilon U^{(1)}\left(\bar{x}_{k}, x_{k}\right)+\ldots,
\end{aligned}
$$

where all two-scale functions $\{\bullet\}^{(0)},\{\bullet\}^{(1)}$ e.t.c. are periodic in $x_{i}{ }^{1}$

Starting with the mechanical part of the problem, the combination of (7) 1,3 with (3) and (1) provides the expression for the first term of the strain,

$$
\varepsilon_{i j}^{(0)}=\bar{\varepsilon}_{i j}+\tilde{\varepsilon}_{i j}, \quad \bar{\varepsilon}_{i j}=\frac{1}{2}\left[\frac{\partial \bar{u}_{i}}{\partial \bar{x}_{j}}+\frac{\partial \bar{u}_{j}}{\partial \bar{x}_{i}}\right], \quad \tilde{\varepsilon}_{i j}=\frac{1}{2}\left[\frac{\partial u_{i}^{(1)}}{\partial x_{j}}+\frac{\partial u_{j}^{(1)}}{\partial x_{i}}\right] .
$$

Due to the periodicity of $u_{i}^{(1)}$, application of the divergence theorem yields $\left\langle\varepsilon_{i j}^{(0)}\right\rangle=\bar{\varepsilon}_{i j}$. Combining (2) with (7) 4 and (1) leads to the expanded form of the equilibrium equation,

$$
\frac{1}{\epsilon} \frac{\partial \sigma_{i j}^{(0)}}{\partial x_{j}}+\frac{\partial \sigma_{i j}^{(0)}}{\partial \bar{x}_{j}}+\frac{\partial \sigma_{i j}^{(1)}}{\partial x_{j}}+\epsilon \ldots=0
$$

\footnotetext{
${ }^{1}$ The assumption that the first expanding term of the displacement vector and the temperature depend only on the macrocoordinates can be considered reasonable in the periodic homogenization theory. In fact, in the cases of thermoelasticity (Ene, 1983; Kalamkarov and Kolpakov, 1997) and thermoviscoelasticity (Yu and Fish, 2002) it is proven that these terms cannot depend on the microscale coordinates. One could also prove this independence without considering apriori connection between stress and strain and between heat flux and temperature gradient by following an approach analogous to the one proposed in Temizer (2012) for large deformation processes.
} 
Following the classical arguments of the periodic homogenization, the $\epsilon^{-1}$ term gives the unit cell equation

$$
\frac{\partial \sigma_{i j}^{(0)}}{\partial x_{j}}=0
$$

while the averaging (over the unit cell) of the $\epsilon^{0}$ term, along with the periodicity of $\sigma_{i j}^{(1)}$ yields

$$
\frac{\partial \bar{\sigma}_{i j}}{\partial \bar{x}_{j}}=0, \quad \bar{\sigma}_{i j}=\left\langle\sigma_{i j}^{(0)}\right\rangle
$$

For the stress $\sigma_{i j}^{(0)}$ and the strain $\varepsilon_{i j}^{(0)}$ it can be shown that the Hill-Mandel principle holds under periodicity conditions. Proof. With the help of equation (8), the average of the product between $\sigma_{i j}^{(0)}$ and $\varepsilon_{i j}^{(0)}$ over the unit cell is written

$$
\left\langle\sigma_{i j}^{(0)} \varepsilon_{i j}^{(0)}\right\rangle=\left\langle\sigma_{i j}^{(0)}\right\rangle \bar{\varepsilon}_{i j}+\left\langle\sigma_{i j}^{(0)} \frac{\partial u_{i}^{(1)}}{\partial x_{j}}\right\rangle=\bar{\sigma}_{i j} \bar{\varepsilon}_{i j}+\left\langle\frac{\partial\left(u_{i}^{(1)} \sigma_{i j}^{(0)}\right)}{\partial x_{j}}\right\rangle-\left\langle u_{i}^{(1)} \frac{\partial \sigma_{i j}^{(0)}}{\partial x_{j}}\right\rangle .
$$

The third term is zero due to (10). Applying the divergence theorem and considering the periodicity of all variables in the microscale, the second term vanishes too. Thus

$$
\left\langle\sigma_{i j}^{(0)} \varepsilon_{i j}^{(0)}\right\rangle=\bar{\sigma}_{i j} \bar{\varepsilon}_{i j}
$$

Similar result holds also for the products $\sigma_{i j}^{(0)} \dot{\varepsilon}_{i j}^{(0)}$ and $\varepsilon_{i j}^{(0)} \dot{\sigma}_{i j}^{(0)}$.

Following analogous steps for the thermal part of the problem, the combination of $(7)_{2,5}$ with (5) and (1) provides the expression for the first term of the temperature gradient,

$$
\nabla \theta_{i}^{(0)}=\bar{\nabla}_{i}+\widetilde{\nabla \theta}_{i}, \quad \overline{\nabla \theta}_{i}=\frac{\partial \bar{\theta}}{\partial \bar{x}_{i}}, \quad \widetilde{\nabla \theta}_{i}=\frac{\partial \theta^{(1)}}{\partial x_{i}}
$$

Due to the periodicity of $\theta_{i}^{(1)}$, application of the divergence theorem leads to $\left\langle\nabla \theta_{i}^{(0)}\right\rangle=\overline{\nabla \theta}_{i}$. Combining (4) with $(7)_{3,4,6,8}$ and (1) yields

$$
\frac{1}{\epsilon} \frac{\partial q_{i}^{(0)}}{\partial x_{i}}+\frac{\partial q_{i}^{(0)}}{\partial \bar{x}_{i}}+\frac{\partial q_{i}^{(1)}}{\partial x_{i}}-\left[\sigma_{i j}^{(0)} \dot{\varepsilon}_{i j}^{(0)}-\dot{U}^{(0)}\right]+\epsilon \ldots=0 .
$$

Following the classical arguments of the periodic homogenization, the $\epsilon^{-1}$ term gives the unit cell equation

$$
\frac{\partial q_{i}^{(0)}}{\partial x_{i}}=0
$$

while the averaging (over the unit cell) of the $\epsilon^{0}$ term, along with the periodicity of $q_{i}^{(1)}$ and the Hill-Mandel condition (12), yields

$$
\frac{\partial \bar{q}_{i}}{\partial \bar{x}_{i}}=\bar{\sigma}_{i j} \dot{\bar{\varepsilon}}_{i j}-\dot{\bar{U}}, \quad \bar{q}_{i}=\left\langle q_{i}^{(0)}\right\rangle, \quad \bar{U}=\left\langle U^{(0)}\right\rangle .
$$

For the heat flux $q_{i}^{(0)}$ and the temperature gradient $\nabla \theta_{i}^{(0)}$ an analogous Hill-Mandel principle holds. 
Proof. With the help of equation (13), the average of the product between $q_{i}^{(0)}$ and $\nabla \theta_{i}^{(0)}$ is written

$$
\left\langle q_{i}^{(0)} \nabla \theta_{i}^{(0)}\right\rangle=\left\langle q_{i}^{(0)}\right\rangle \bar{\nabla}_{i}+\left\langle q_{i}^{(0)} \frac{\partial \theta^{(1)}}{\partial x_{i}}\right\rangle=\bar{q}_{i} \overline{\nabla \theta}_{i}+\left\langle\frac{\partial\left(\theta^{(1)} q_{i}^{(0)}\right)}{\partial x_{i}}\right\rangle-\left\langle\theta^{(1)} \frac{\partial q_{i}^{(0)}}{\partial x_{i}}\right\rangle .
$$

The third term is zero due to (15). Applying the divergence theorem and considering the periodicity of all variables in the microscale, the second term vanishes too. Thus

$$
\left\langle q_{i}^{(0)} \nabla \theta_{i}^{(0)}\right\rangle=\bar{q}_{i} \overline{\nabla \theta}_{i}
$$

Finally, the second law of thermodynamics (6) is written

$$
\bar{\theta} \dot{s}^{(0)}+\sigma_{i j}^{(0)} \dot{\varepsilon}_{i j}^{(0)}-\dot{U}^{(0)}-\frac{1}{\bar{\theta}+\epsilon \theta^{(1)}+\ldots} q_{i}^{(0)} \nabla \theta_{i}^{(0)}+\epsilon \ldots \geq 0 .
$$

For $\epsilon \rightarrow 0$, the microscale dissipation inequality is obtained,

$$
\bar{\theta} \dot{s}^{(0)}+\sigma_{i j}^{(0)} \dot{\varepsilon}_{i j}^{(0)}-\dot{U}^{(0)}-\frac{1}{\bar{\theta}} q_{i}^{(0)} \nabla \theta_{i}^{(0)} \geq 0 .
$$

Averaging this expression and taking into account (12), (17) and (16) 4 yields

$$
\bar{\theta} \dot{\bar{s}}+\bar{\sigma}_{i j} \dot{\bar{\varepsilon}}_{i j}-\dot{\bar{U}}-\frac{1}{\bar{\theta}} \bar{q}_{i} \overline{\nabla \theta}_{i} \geq 0, \quad \bar{s}=\left\langle s^{(0)}\right\rangle .
$$

It is worth mentioning that the obtained results are valid, under certain conditions, in the case where body forces, inertia forces and heat radiation sources are taken into account (see for instance Sanchez-Palencia, 1978; Ene, 1983).

\subsection{Energy potentials and dissipation}

When considering a specific type of material, the internal energy $U^{\epsilon}$ needs to be expressed in terms of certain variables that dictate the status of the material at each time instant. Apart from the strain and the entropy, a set of internal variables could be necessary to describe the material state. Thus, one can choose an internal energy of the general form

$$
U^{\epsilon}:=U^{\epsilon}\left(\varepsilon_{i j}^{\epsilon}, s^{\epsilon}, \zeta_{\alpha}^{\epsilon}\right)
$$

where $\zeta_{\alpha}^{\epsilon}$ for simplicity denotes the list of all scalars $z^{\epsilon}$, vectors $z_{i}^{\epsilon}$ and second order tensors $z_{i j}^{\epsilon}$ internal variables of the composite material (i.e. of all constituents that appear inside the composite). In the sequel the following operator is introduced:

$$
\frac{\partial\{\bullet\}}{\partial \zeta_{\alpha}} \dot{\zeta}_{\alpha}=\sum_{m} \frac{\partial\{\bullet\}}{\partial z^{(m)}} \dot{z}^{(m)}+\sum_{n} \frac{\partial\{\bullet\}}{\partial z_{i}^{(n)}} \dot{z}_{i}^{(n)}+\sum_{q} \frac{\partial\{\bullet\}}{\partial z_{i j}^{(q)}} \dot{z}_{i j}^{(q)} .
$$

Identifying constitutive laws in terms of entropy is not practical, so it is more convenient to introduce alternative potentials. The Helmholtz and Gibbs free energy potentials are written as

$$
\Psi^{\epsilon}:=\Psi^{\epsilon}\left(\varepsilon_{i j}^{\epsilon}, \theta^{\epsilon}, \zeta_{\alpha}^{\epsilon}\right), \quad G^{\epsilon}:=G^{\epsilon}\left(\sigma_{i j}^{\epsilon}, \theta^{\epsilon}, \zeta_{\alpha}^{\epsilon}\right)
$$


which are connected with the internal energy through the relations

$$
\Psi^{\epsilon}=U^{\epsilon}-s^{\epsilon} \theta^{\epsilon}, \quad G^{\epsilon}=U^{\epsilon}-s^{\epsilon} \theta^{\epsilon}-\sigma_{i j}^{\epsilon} \varepsilon_{i j}^{\epsilon} .
$$

According to the classical approach and assumptions of thermodynamics, the following relations are obtained:

$$
\sigma_{i j}^{\epsilon}=\frac{\partial \Psi^{\epsilon}}{\partial \varepsilon_{i j}^{\epsilon}}, \quad s^{\epsilon}=-\frac{\partial \Psi^{\epsilon}}{\partial \theta^{\epsilon}}, \quad \text { or } \quad \varepsilon_{i j}^{\epsilon}=-\frac{\partial G^{\epsilon}}{\partial \sigma_{i j}^{\epsilon}}, \quad s^{\epsilon}=-\frac{\partial G^{\epsilon}}{\partial \theta^{\epsilon}},
$$

while the second law takes the form

$$
\gamma_{\mathrm{loc}}^{\epsilon}-\frac{1}{\theta^{\epsilon}} q_{i}^{\epsilon} \nabla \theta_{i}^{\epsilon} \geq 0, \quad \gamma_{\mathrm{loc}}^{\epsilon}=-\frac{\partial \Psi^{\epsilon}}{\partial \zeta_{\alpha}^{\epsilon}} \dot{\zeta}_{\alpha}^{\epsilon}, \quad \text { or } \quad \gamma_{\mathrm{loc}}^{\epsilon}=-\frac{\partial G^{\epsilon}}{\partial \zeta_{\alpha}^{\epsilon}} \dot{\zeta}_{\alpha}^{\epsilon},
$$

where $\gamma_{\text {loc }}$ denotes local dissipation due to mechanical work. Thus, the rate of the Helmholtz free energy potential is expressed as

$$
\dot{\Psi}^{\epsilon}=\sigma_{i j}^{\epsilon} \dot{\varepsilon}_{i j}^{\epsilon}-s^{\epsilon} \dot{\theta}^{\epsilon}+\frac{\partial \Psi^{\epsilon}}{\partial \zeta_{\alpha}^{\epsilon}} \dot{\zeta}_{\alpha}^{\epsilon}=\sigma^{\epsilon} \dot{\varepsilon}_{i j}^{\epsilon}-s^{\epsilon} \dot{\theta}^{\epsilon}-\gamma_{\mathrm{loc}}^{\epsilon},
$$

and the rate of the Gibbs free energy potential is written

$$
\dot{G}^{\epsilon}=-\varepsilon_{i j}^{\epsilon} \dot{\sigma}_{i j}^{\epsilon}-s^{\epsilon} \dot{\theta}^{\epsilon}+\frac{\partial G^{\epsilon}}{\partial \zeta_{\alpha}^{\epsilon}} \dot{\zeta}_{\alpha}^{\epsilon}=-\varepsilon^{\epsilon} \dot{\sigma}_{i j}^{\epsilon}-s^{\epsilon} \dot{\theta}^{\epsilon}-\gamma_{\mathrm{loc}}^{\epsilon} .
$$

Utilizing the same technique as in the previous subsection, the following variables are considered to follow an asymptotic series expansion:

$$
\begin{aligned}
\Psi^{\epsilon}\left(x_{k}^{\epsilon}\right) & =\Psi^{(0)}\left(\bar{x}_{k}, x_{k}\right)+\epsilon \Psi^{(1)}\left(\bar{x}_{k}, x_{k}\right)+\ldots, \\
G^{\epsilon}\left(x_{k}^{\epsilon}\right) & =G^{(0)}\left(\bar{x}_{k}, x_{k}\right)+\epsilon G^{(1)}\left(\bar{x}_{k}, x_{k}\right)+\ldots, \\
-\frac{\partial \Psi^{\epsilon}}{\partial \zeta_{\alpha}^{\epsilon}}\left(x_{k}^{\epsilon}\right) & =-\left(\frac{\partial \Psi}{\partial \zeta_{\alpha}}\right)^{(0)}\left(\bar{x}_{k}, x_{k}\right)-\epsilon\left(\frac{\partial \Psi}{\partial \zeta_{\alpha}}\right)^{(1)}\left(\bar{x}_{k}, x_{k}\right)+\ldots, \\
-\frac{\partial G^{\epsilon}}{\partial \zeta_{\alpha}^{\epsilon}}\left(x_{k}^{\epsilon}\right) & =-\left(\frac{\partial G}{\partial \zeta_{\alpha}}\right)^{(0)}\left(\bar{x}_{k}, x_{k}\right)-\epsilon\left(\frac{\partial G}{\partial \zeta_{\alpha}}\right)^{(1)}\left(\bar{x}_{k}, x_{k}\right)+\ldots, \\
\zeta_{\alpha}^{\epsilon}\left(x_{k}^{\epsilon}\right) & =\zeta_{\alpha}^{(0)}\left(\bar{x}_{k}, x_{k}\right)+\epsilon \zeta_{\alpha}^{(1)}\left(\bar{x}_{k}, x_{k}\right)+\ldots \\
\gamma_{\mathrm{loc}}^{\epsilon}\left(x_{k}^{\epsilon}\right) & =\gamma_{\mathrm{loc}}^{(0)}\left(\bar{x}_{k}, x_{k}\right)+\epsilon \gamma_{\mathrm{loc}}^{(1)}\left(\bar{x}_{k}, x_{k}\right)+\ldots
\end{aligned}
$$

where all two-scale functions are periodic in $x_{i}$. Then, for $\epsilon \rightarrow 0$, equations (24), (27), (28), combined with (7), provide the following expressions in the microscale:

$$
\begin{gathered}
\Psi^{(0)}=U^{(0)}-s^{(0)} \bar{\theta}, \quad G^{(0)}=U^{(0)}-s^{(0)} \bar{\theta}-\sigma_{i j}^{(0)} \varepsilon_{i j}^{(0)}, \\
\dot{\Psi}(0)=\sigma_{i j}^{(0)} \dot{\varepsilon}_{i j}^{(0)}-s^{(0)} \dot{\bar{\theta}}+\left(\frac{\partial \Psi}{\partial \zeta_{\alpha}}\right)^{(0)} \dot{\zeta}_{\alpha}^{(0)}=\sigma_{i j}^{(0)} \dot{\varepsilon}_{i j}^{(0)}-s^{(0)} \dot{\bar{\theta}}-\gamma_{\mathrm{loc}}^{(0)} \\
\dot{G}^{(0)}=-\varepsilon_{i j}^{(0)} \dot{\sigma}_{i j}^{(0)}-s^{(0)} \dot{\bar{\theta}}+\left(\frac{\partial G}{\partial \zeta_{\alpha}}\right)^{(0)} \dot{\zeta}_{\alpha}^{(0)}=-\varepsilon_{i j}^{(0)} \dot{\sigma}_{i j}^{(0)}-s^{(0)} \dot{\bar{\theta}}-\gamma_{\mathrm{loc}}^{(0)}
\end{gathered}
$$

From these expressions it becomes clear that

$$
\Psi^{(0)}:=\Psi^{(0)}\left(\varepsilon_{i j}^{(0)}, \bar{\theta}, \zeta_{\alpha}^{(0)}\right), \quad \sigma_{i j}^{(0)}=\frac{\partial \Psi^{(0)}}{\partial \varepsilon_{i j}^{(0)}}, \quad s^{(0)}=-\frac{\partial \Psi^{(0)}}{\partial \bar{\theta}}, \quad-\left(\frac{\partial \Psi}{\partial \zeta_{\alpha}}\right)^{(0)}=-\frac{\partial \Psi^{(0)}}{\partial \zeta_{\alpha}^{(0)}}, \quad \gamma_{\mathrm{loc}}^{(0)}=-\frac{\partial \Psi^{(0)}}{\partial \zeta_{\alpha}^{(0)}} \dot{\zeta}_{\alpha}^{(0)},
$$


and

$$
G^{(0)}:=G^{(0)}\left(\sigma_{i j}^{(0)}, \bar{\theta}, \zeta_{\alpha}^{(0)}\right), \quad \varepsilon_{i j}^{(0)}=-\frac{\partial G^{(0)}}{\partial \sigma_{i j}^{(0)}}, \quad s^{(0)}=-\frac{\partial G^{(0)}}{\partial \bar{\theta}}, \quad-\left(\frac{\partial G}{\partial \zeta_{\alpha}}\right)^{(0)}=-\frac{\partial G^{(0)}}{\partial \zeta_{\alpha}^{(0)}}, \quad \gamma_{\mathrm{loc}}^{(0)}=-\frac{\partial G^{(0)}}{\partial \zeta_{\alpha}^{(0)}} \dot{\zeta}_{\alpha}^{(0)}
$$

As it can be observed from the Helmholtz free energy related expression $(31)_{2}$, the microstress depends on the macrotemperature (since $\Psi^{(0)}$ is a function of $\bar{\theta}$ ). This result is compatible with the one obtained in linear thermoelasticity (Ene, 1983; Kalamkarov and Kolpakov, 1997). Averaging equations (30) over the unit cell and considering $(16)_{3},(12)$, yields

$$
\begin{gathered}
\bar{\Psi}=\bar{U}-\bar{s} \bar{\theta}, \quad \bar{\Psi}=\left\langle\Psi^{(0)}\right\rangle, \\
\dot{\bar{\Psi}}=\bar{\sigma}_{i j} \dot{\bar{\varepsilon}}_{i j}-\bar{s} \dot{\bar{\theta}}+\left\langle\frac{\partial \Psi^{(0)}}{\partial \zeta_{\alpha}^{(0)}} \dot{\zeta}_{\alpha}^{(0)}\right\rangle=\bar{\sigma}_{i j} \dot{\bar{\varepsilon}}_{i j}-\overline{\bar{s}} \dot{\bar{\theta}}-\bar{\gamma}_{\mathrm{loc}}, \quad \bar{\gamma}_{\mathrm{loc}}=\left\langle\gamma_{\mathrm{loc}}^{(0)}\right\rangle, \\
\bar{G}=\bar{U}-\bar{s} \bar{\theta}-\bar{\sigma}_{i j} \bar{\varepsilon}_{i j}, \quad \bar{G}=\left\langle G^{(0)}\right\rangle, \\
\dot{\bar{G}}=-\bar{\varepsilon}_{i j} \dot{\bar{\sigma}}_{i j}-\bar{s} \dot{\bar{\theta}}+\left\langle\frac{\partial G^{(0)}}{\partial \zeta_{\alpha}^{(0)}} \dot{\zeta}_{\alpha}^{(0)}\right\rangle=-\bar{\varepsilon}_{i j} \dot{\bar{\sigma}}_{i j}-\overline{\bar{s}} \dot{\bar{\theta}}-\bar{\gamma}_{\mathrm{loc}}, \quad \bar{\gamma}_{\mathrm{loc}}=\left\langle\gamma_{\mathrm{loc}}^{(0)}\right\rangle .
\end{gathered}
$$

Tables 1 and 2 present a summary of the obtained variables and expressions in both scales.

Table 1: Microscale and macroscale variables

\begin{tabular}{|c|c|c|}
\hline variable & microscale & macroscale \\
\hline strain & $\varepsilon_{i j}^{(0)}$ & $\bar{\varepsilon}_{i j}=\left\langle\varepsilon_{i j}^{(0)}\right\rangle$ \\
\hline stress & $\sigma_{i j}^{(0)}$ & $\bar{\sigma}_{i j}=\left\langle\sigma_{i j}^{(0)}\right\rangle$ \\
\hline temperature gradient & $\nabla \theta_{i}^{(0)}$ & $\bar{\nabla} \theta_{i}=\left\langle\nabla \theta_{i}^{(0)}\right\rangle$ \\
\hline heat flux & $q_{i}^{(0)}$ & $\bar{q}_{i}=\left\langle q_{i}^{(0)}\right\rangle$ \\
\hline specific entropy per unit volume & $s^{(0)}$ & $\bar{s}=\left\langle s^{(0)}\right\rangle$ \\
\hline internal energy per unit volume & $U^{(0)}$ & $\bar{U}=\left\langle U^{(0)}\right\rangle$ \\
\hline Helmholtz free energy potential & $\Psi^{(0)}\left(\varepsilon_{i j}^{(0)}, \bar{\theta}, \zeta_{\alpha}^{(0)}\right)$ & $\bar{\Psi}=\left\langle\Psi^{(0)}\right\rangle$ \\
\hline Gibbs free energy potential & $G^{(0)}\left(\sigma_{i j}^{(0)}, \bar{\theta}, \zeta_{\alpha}^{(0)}\right)$ & $\bar{G}=\left\langle G^{(0)}\right\rangle$ \\
\hline local dissipation & $\gamma_{\mathrm{loc}}^{(0)}=-\frac{\partial \Psi^{(0)}}{\partial \zeta_{\alpha}^{(0)}} \dot{\zeta}_{\alpha}^{(0)} \quad$ or $\gamma_{\mathrm{loc}}^{(0)}=-\frac{\partial G^{(0)}}{\partial \zeta_{\alpha}^{(0)}} \dot{\zeta}_{\alpha}^{(0)}$ & $\bar{\gamma}_{\mathrm{loc}}=\left\langle\gamma_{\mathrm{loc}}^{(0)}\right\rangle$ \\
\hline
\end{tabular}


Table 2: Microscale and macroscale equations

\begin{tabular}{|c|c|c|}
\hline equation & microscale & macroscale \\
\hline kinematics & $\varepsilon_{i j}^{(0)}=\bar{\varepsilon}_{i j}+\frac{1}{2}\left[\frac{\partial u_{i}^{(1)}}{\partial x_{j}}+\frac{\partial u_{j}^{(1)}}{\partial x_{i}}\right]$ & $\bar{\varepsilon}_{i j}=\frac{1}{2}\left[\frac{\partial \bar{u}_{i}}{\partial \bar{x}_{j}}+\frac{\partial \bar{u}_{j}}{\partial \bar{x}_{i}}\right]$ \\
\hline equilibrium & $\frac{\partial \sigma_{i j}^{(0)}}{\partial x_{j}}=0$ & $\frac{\partial \bar{\sigma}_{i j}}{\partial \bar{x}_{j}}=0$ \\
\hline temperature gradient & $\nabla \theta_{i}^{(0)}=\bar{\nabla}_{i}+\frac{\partial \theta^{(1)}}{\partial x_{i}}$ & $\bar{\nabla}_{i}=\frac{\partial \bar{\theta}}{\partial \bar{x}_{i}}$ \\
\hline energy equation & $\frac{\partial q_{i}^{(0)}}{\partial x_{i}}=0$ & $\frac{\partial \bar{q}_{i}}{\partial \bar{x}_{i}}=\bar{\sigma}_{i j} \dot{\bar{\varepsilon}}_{i j}-\dot{\bar{U}}$ \\
\hline dissipation inequality & $\bar{\theta} \dot{s}^{(0)}+\sigma_{i j}^{(0)} \dot{\varepsilon}_{i j}^{(0)}-\dot{U}^{(0)}-\frac{1}{\bar{\theta}} q_{i}^{(0)} \nabla \theta_{i}^{(0)} \geq 0$ & $\bar{\theta} \dot{\bar{s}}+\bar{\sigma}_{i j} \dot{\bar{\varepsilon}}_{i j}-\dot{\bar{U}}-\frac{1}{\bar{\theta}} \bar{q}_{i} \bar{\nabla}_{i} \geq 0$ \\
\hline Helmholtz-internal energy relation & $\Psi^{(0)}=U^{(0)}-s^{(0)} \bar{\theta}$ & $\bar{\Psi}=\bar{U}-\bar{s} \bar{\theta}$ \\
\hline rate of Helmholtz free energy & $\dot{\Psi}^{(0)}=\sigma_{i j}^{(0)} \dot{\varepsilon}_{i j}^{(0)}-s^{(0)} \dot{\bar{\theta}}+\frac{\partial \Psi^{(0)}}{\partial \zeta_{\alpha}^{(0)}} \dot{\zeta}_{\alpha}^{(0)}$ & $\dot{\bar{\Psi}}=\bar{\sigma}_{i j} \dot{\bar{\varepsilon}}_{i j}-\overline{\bar{s} \theta}-\bar{\gamma}_{\mathrm{loc}}$ \\
\hline Gibbs-internal energy relation & $G^{(0)}=U^{(0)}-s^{(0)} \bar{\theta}-\sigma_{i j}^{(0)} \varepsilon_{i j}^{(0)}$ & $\bar{G}=\bar{U}-\bar{s} \bar{\theta}-\bar{\sigma}_{i j} \bar{\varepsilon}_{i j}$ \\
\hline rate of Gibbs free energy & $\dot{G}^{(0)}=-\varepsilon_{i j}^{(0)} \dot{\sigma}_{i j}^{(0)}-s^{(0)} \dot{\bar{\theta}}+\frac{\partial G^{(0)}}{\partial \zeta_{\alpha}^{(0)}} \dot{\zeta}_{\alpha}^{(0)}$ & $\dot{\bar{G}}=-\bar{\varepsilon}_{i j} \dot{\bar{\sigma}}_{i j}-\overline{\bar{s}} \dot{\bar{\theta}}-\bar{\gamma}_{\mathrm{loc}}$ \\
\hline
\end{tabular}


At this point, two issues are important to be discussed:

1. Combining equations (19), (20), (30) 1 and (33) $)_{1}$, the second law of thermodynamics, in terms of the Helmholtz free energy, is written for both microscale and macroscale as

$$
\dot{\bar{\theta}} s^{(0)}+\sigma_{i j}^{(0)} \dot{\varepsilon}_{i j}^{(0)}-\dot{\Psi}^{(0)}-\frac{1}{\bar{\theta}} q_{i}^{(0)} \nabla \theta_{i}^{(0)} \geq 0, \quad \dot{\bar{\theta}} \bar{s}+\bar{\sigma}_{i j} \dot{\bar{\varepsilon}}_{i j}-\dot{\bar{\Psi}}-\frac{1}{\bar{\theta}} \bar{q}_{i} \bar{\nabla}_{i} \geq 0
$$

The fourth term of equation $(34)_{1}$ can be always non-negative once a Fourier-law type for the heat flux vector is considered, i.e.

$$
q_{i}^{(0)}=-\kappa_{i j} \nabla \theta_{j}^{(0)}
$$

with positive definite thermal conductivity tensor $\kappa_{i j}$. Since the second expression of (34) arises from the first expression by averaging, $-\frac{1}{\bar{\theta}} \bar{q}_{i} \bar{\nabla}_{i}$ is also non-negative. By decoupling the two dissipative mechanisms (due to mechanical work and due to heat conduction), one can have a stronger form of the dissipation inequality for both scales,

$$
\dot{\bar{\theta}} s^{(0)}+\sigma_{i j}^{(0)} \dot{\varepsilon}_{i j}^{(0)}-\dot{\Psi}^{(0)} \geq 0, \quad \dot{\bar{\theta}} \bar{s}+\bar{\sigma}_{i j} \dot{\bar{\varepsilon}}_{i j}-\dot{\bar{\Psi}} \geq 0
$$

Moreover, according to $(31)_{1}$, the microscopic Helmholtz free energy is a function of the macroscopic temperature. These observations eventually indicate that the unit cell problem (10) needs to be solved under uniform (macroscopic) temperature conditions, in order to ensure that the expression (36) $)_{1}$ and its average (36) 2 are both satisfied. The same conclusions are also derived when utilizing the Gibbs free energy potential.

2. While the internal variables and their conjugate thermodynamic forces are well defined in the microscale, this is not true for the macroscale problem. The averaged term of $(33)_{2}$ (or $\left.(33)_{4}\right)$ cannot be decomposed into a macroscopic variables product, like the rest of the terms. Only the average of $\gamma_{\text {loc }}^{(0)}$ can be identified as the macroscopic $\bar{\gamma}_{\text {loc }}$ (a discussion about the implications that arise from this product can be found in Tsalis et al., 2015). Thus, in the general case, the mechanical dissipation in the macroscale can be obtained only by averaging the microscale mechanical dissipation over the unit cell. This causes problems in expressing analytically the macroscopic energy equation (16) $)_{1}$. One way to overcome this difficulty is to consider a linearized, incremental form of the actual thermomechanical problem.

\section{Linearized, incremental form of the homogenization problem}

In many dissipative materials, the stress is related with the strain, the temperature and the internal variables in a complicated way, where also time derivatives are involved. In computational, incremental formulations for such laws, it is customary to first linearize the actual problem in time and then proceed to a second linearization of the nonlinear expressions. In the current work, an approach based on the well known return mapping algorithm scheme is utilized (Simo and Hughes, 1998). Similar numerical homogenization techniques have been proposed in the literature for elastoplastic (Terada and Kikuchi, 2001; Asada and Ohno, 2007; Tsalis et al., 2013), magnetoelastic (Javili et al., 
2013) and shape memory alloy composites (Chatzigeorgiou et al., 2015). In brief, the two problems (macroscale and unit cell) are solved simultaneously, using an iterative scheme (Figure 1): from the macroscale analysis the macroscopic strains, temperatures and tamperature gradients are obtained, which are used in the unit cell problem to compute the microscale variables and the rest of the unknown macroscopic variables (stresses, heat fluxes e.t.c.). Moreover, the unit cell problem provides information to compute the macroscopic tangent moduli, which are required in the macroscale analysis.

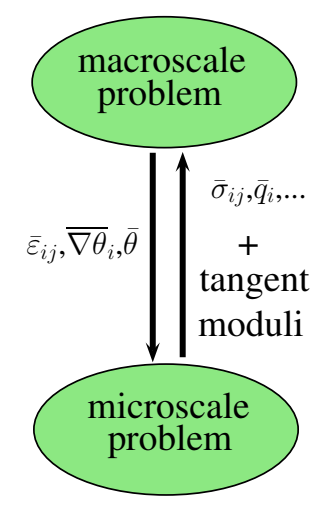

Figure 1: Computational homogenization scheme.

\subsection{Preliminaries of linearized, incremental formulation}

The computational framework includes two types of increments of a quantity $x$ : i) increment in time, denoted by $\Delta x$ and ii) increment during the Newton-Raphson scheme in the linearized equations, denoted by the symbol $\delta x$.

In a backward Euler fully implicit numerical scheme, the value of a given quantity $x$ is updated from the previous time step $n$ to the current $n+1$ per

$$
x^{(n+1)}=x^{(n)}+\Delta x^{(n+1)} .
$$

The time derivative of a quantity $x$ is computed by the relation

$$
\dot{x}^{(n+1)} \Delta t=\Delta x^{(n+1)}, \text { with } \Delta t=t^{(n+1)}-t^{(n)}
$$

In the second step of linearization an iterative procedure is required. In this procedure, the current value is updated from iteration $k$ to iteration $k+1$ per

$$
x^{(n+1)(k+1)}=x^{(n+1)(k)}+\delta x^{(n+1)(k)} \text { or } \Delta x^{(n+1)(k+1)}=\Delta x^{(n+1)(k)}+\delta x^{(n+1)(k)} .
$$

Additionally, the time derivative of a quantity $x$ is numerically discretized according to the relation

$$
\dot{x}^{(n+1)(k)} \Delta t=x^{(n+1)(k)}-x^{(n)}=\Delta x^{(n+1)(k)} .
$$


In this formalism, the iterational increment of a function $f(x, \dot{x})$ is written as

$$
\delta f^{(n+1)(k)}=\left[\frac{\partial f}{\partial x}\right]^{(n+1)(k)} \delta x^{(n+1)(k)}+\frac{1}{\Delta t}\left[\frac{\partial f}{\partial \dot{x}}\right]^{(n+1)(k)} \delta x^{(n+1)(k)} .
$$

In order to simplify the expressions, the superscripts are omitted in the sequel. Any quantity $x$ denotes the $x^{(n+1)(k)}$, the increment $\Delta x$ denotes the $\Delta x^{(n+1)(k)}$, while the increment $\delta x$ denotes the $\delta x^{(n+1)(k)}$.

\subsection{Unit cell problem}

The equations of the unit cell problem $(8)_{1},(13)_{1},(10),(15)$ are written in the linearized, incremental form as

$$
\begin{gathered}
\delta \varepsilon_{i j}^{(0)}=\delta \bar{\varepsilon}_{i j}+\frac{1}{2}\left[\frac{\partial \delta u_{i}^{(1)}}{\partial x_{j}}+\frac{\partial \delta u_{j}^{(1)}}{\partial x_{i}}\right], \quad \delta \nabla \theta_{i}^{(0)}=\delta \overline{\nabla \theta}_{i}+\frac{\partial \delta \theta^{(1)}}{\partial x_{i}}, \\
\frac{\partial}{\partial x_{j}}\left(\sigma_{i j}^{(0)}+\delta \sigma_{i j}^{(0)}\right)=0, \quad \frac{\partial}{\partial x_{i}}\left(q_{i}^{(0)}+\delta q_{i}^{(0)}\right)=0 .
\end{gathered}
$$

These equations are accompanied by appropriate constitutive laws for the increments of the stress tensor $\sigma_{i j}^{(0)}$ and the quantity

$$
r^{(0)}=\sigma_{i j}^{(0)} \dot{\varepsilon}_{i j}^{(0)}-\dot{U}^{(0)}
$$

Recall that these quantities depend on the microscopic Helmholtz energy potential, which is a function of the strain $\varepsilon_{i j}^{(0)}$, the temperature $\bar{\theta}$ and suitable internal variables. In numerical schemes, the increments of the internal variables can be expressed in terms of the increments of strain and temperature. Thus, the linearized constitutive expressions can eventually take the forms

$$
\delta \sigma_{i j}^{(0)}=D_{i j k l}^{\varepsilon} \delta \varepsilon_{k l}^{(0)}+D_{i j}^{\theta} \delta \bar{\theta}, \quad \delta r^{(0)}=R_{i j}^{\varepsilon} \delta \varepsilon_{i j}^{(0)}+R^{\theta} \delta \bar{\theta}
$$

where $D_{i j k l}^{\varepsilon}, D_{i j}^{\theta}, R_{i j}^{\varepsilon}, R^{\theta}$ are assumed constant during the iteration step. In Appendix A the analytical expressions of these tensors per incremental step are presented for several constitutive laws. An important limitation is that the fourth order tensor $D_{i j k l}^{\varepsilon}$ needs to be positive definite at all times, in order to ensure unique solution of the linearized system. Finally, the increment of the heat flux vector can be described in terms of the temperature gradient vector by a linear law of the form

$$
\delta q_{i}^{(0)}=-\kappa_{i j} \delta \nabla \theta_{j}^{(0)}
$$

where the thermal conductivity tensor $\kappa_{i j}$ is considered positive definite and is assumed constant during the iteration step. With regard to boundary conditions, periodicity of $\delta u_{i}^{(1)}, \delta \theta^{(1)}$ and antiperiodicity of $\delta \sigma_{i j}^{(0)} n_{j}, \delta q_{i}^{(0)} n_{i}$ are imposed. From the linearized system (37), (39) and (40), two types of information are required: a) the macroscopic fields and b) appropriate macroscopic quantities of $D_{i j k l}^{\varepsilon}, D_{i j}^{\theta}, R_{i j}^{\varepsilon}, R^{\theta}$ and $\kappa_{i j}$, which are essential for the solution of the macroscopic problem. To obtain both information, a two-step approach is followed: 
1. At the first step, the macrostrain tensor, the macrotemperature and the macrotemperature gradient are provided exclusively by the macroscale analysis (presented later in this section), which means that they do not iterate during the calculations, and thus the terms $\delta \bar{\varepsilon}_{i j}, \delta \bar{\theta}$ and $\delta \overline{\nabla \theta}_{i}$ are set to zero. Then $(37)_{3,4}$ are reduced to

$$
\frac{\partial}{\partial x_{j}}\left(\sigma_{i j}^{(0)}+D_{i j k l}^{\varepsilon} \frac{\partial \delta u_{k}^{(1)}}{\partial x_{l}}\right)=0, \quad \frac{\partial}{\partial x_{i}}\left(q_{i}^{(0)}-\kappa_{i j} \frac{\partial \delta \theta^{(1)}}{\partial x_{j}}\right)=0
$$

which can be solved iteratively. When the convergence is achieved (i.e. when $\delta u_{i}^{(1)}$ and $\delta \theta^{(1)}$ are almost zero), the time increments and the actual values of the fluctuating terms $u_{i}^{(1)}$ and $\theta^{(1)}$ are evaluated, allowing all the microscopic quantities and their averaged (macroscopic) counterparts of Table 1 to be computed.

2. Once the first step is completed, the residual terms are assumed exactly zero. The next step is to obtain the macroscopic tangent moduli that permit to perform FE calculations in the macroscale analysis. Thus, technically the terms $\delta \bar{\varepsilon}_{i j}, \delta \bar{\theta}$ and $\delta \bar{\nabla}_{i}$ are 'released' from being zero. With this hypothesis the microscale equations are written as

$$
\frac{\partial}{\partial x_{j}}\left(D_{i j k l}^{\varepsilon} \delta \bar{\varepsilon}_{k l}+D_{i j}^{\theta} \delta \bar{\theta}+D_{i j k l}^{\varepsilon} \frac{\partial \delta u_{k}^{(1)}}{\partial x_{l}}\right)=0, \quad \frac{\partial}{\partial x_{i}}\left(\kappa_{i j} \delta \overline{\nabla \theta}_{j}+\kappa_{i j} \frac{\partial \delta \theta^{(1)}}{\partial x_{j}}\right)=0 .
$$

Note that the microscopic tensors $D_{i j k l}^{\varepsilon}$ and $D_{i j}^{\theta}$ are the last values obtained from the previous step. The solution of the above homogeneous system (up to an arbitrary macroscopic constant) is written in the form (Ene, 1983)

$$
\delta u_{i}^{(1)}=\delta \bar{\varepsilon}_{k l} \chi_{k l i}^{\varepsilon}+\delta \bar{\theta} \chi_{i}^{\theta}, \quad \delta \theta^{(1)}=\delta \bar{\nabla}_{i} \psi_{i}^{\theta},
$$

where the third order tensor $\chi_{k l i}^{\varepsilon}$ and the vectors $\chi_{i}^{\theta}, \psi_{i}^{\theta}$ are called corrector terms. Substituting (43) in (42) yields

$$
\frac{\partial}{\partial x_{j}}\left(D_{i j k l}^{\varepsilon}+D_{i j m n}^{\varepsilon} \frac{\partial \chi_{k l m}^{\varepsilon}}{\partial x_{n}}\right) \delta \bar{\varepsilon}_{k l}+\frac{\partial}{\partial x_{j}}\left(D_{i j}^{\theta}+D_{i j k l}^{\varepsilon} \frac{\partial \chi_{k}^{\theta}}{\partial x_{l}}\right) \delta \bar{\theta}=0, \quad \frac{\partial}{\partial x_{i}}\left(\kappa_{i j}+\kappa_{i k} \frac{\partial \psi_{j}^{\theta}}{\partial x_{k}}\right) \delta \bar{\nabla}_{j}=0
$$

The last two equations are valid for arbitrary macroscale quantities $\delta \bar{\varepsilon}_{i j}, \delta \bar{\theta}$ and $\delta \bar{\nabla} \bar{\theta}_{i}$ only if the corrector terms satisfy the linear equations

$$
\frac{\partial}{\partial x_{j}}\left(D_{i j k l}^{\varepsilon}+D_{i j m n}^{\varepsilon} \frac{\partial \chi_{k l m}^{\varepsilon}}{\partial x_{n}}\right)=0, \quad \frac{\partial}{\partial x_{j}}\left(D_{i j}^{\theta}+D_{i j k l}^{\varepsilon} \frac{\partial \chi_{k}^{\theta}}{\partial x_{l}}\right)=0, \quad \frac{\partial}{\partial x_{i}}\left(\kappa_{i j}+\kappa_{i k} \frac{\partial \psi_{j}^{\theta}}{\partial x_{k}}\right)=0
$$

Using the solution (43), the increments of the other microscopic fields are written as

$$
\begin{gathered}
\delta \varepsilon_{i j}^{(0)}=A_{i j k l}^{\varepsilon} \delta \bar{\varepsilon}_{k l}+A_{i j}^{\theta} \delta \bar{\theta}, \quad \delta \nabla \theta_{i}^{(0)}=\Theta_{i j} \delta \bar{\nabla}_{j} \\
\delta \sigma_{i j}^{(0)}=D_{i j m n}^{\varepsilon} A_{m n k l}^{\varepsilon} \delta \bar{\varepsilon}_{k l}+\left[D_{i j}^{\theta}+D_{i j k l}^{\varepsilon} A_{k l}^{\theta}\right] \delta \bar{\theta}, \quad \delta q_{i}^{(0)}=-\kappa_{i k} \Theta_{k j} \delta \overline{\nabla \theta}_{j} \\
\delta r^{(0)}=R_{k l}^{\varepsilon} A_{k l i j}^{\varepsilon} \delta \bar{\varepsilon}_{i j}+\left[R^{\theta}+R_{i j}^{\varepsilon} A_{i j}^{\theta}\right] \delta \bar{\theta}
\end{gathered}
$$

where

$$
A_{i j k l}^{\varepsilon}=\mathcal{I}_{i j k l}+\frac{1}{2}\left[\frac{\partial \chi_{k l i}^{\varepsilon}}{\partial x_{j}}+\frac{\partial \chi_{k l j}^{\varepsilon}}{\partial x_{i}}\right], \quad A_{i j}^{\theta}=\frac{1}{2}\left[\frac{\partial \chi_{i}^{\theta}}{\partial x_{j}}+\frac{\partial \chi_{j}^{\theta}}{\partial x_{i}}\right], \quad \Theta_{i j}=I_{i j}+\frac{\partial \psi_{j}^{\theta}}{\partial x_{i}}
$$


are strain and temperature gradient related concentration tensors. Moreover, $\mathcal{I}_{i j k l}$ denotes the fourth order symmetric identity tensor and $I_{i j}$ is the second order identity tensor. Averaging of these fields over the unit cell yields the macroscopic tensors

$$
\begin{gathered}
\bar{D}_{i j k l}^{\varepsilon}=\left\langle D_{i j m n}^{\varepsilon} A_{m n k l}^{\varepsilon}\right\rangle, \quad \bar{D}_{i j}^{\theta}=\left\langle D_{i j}^{\theta}+D_{i j k l}^{\varepsilon} A_{k l}^{\theta}\right\rangle, \quad \bar{\kappa}_{i j}=\left\langle\kappa_{i k} \Theta_{k j}\right\rangle, \\
\bar{R}_{i j}^{\varepsilon}=\left\langle R_{k l}^{\varepsilon} A_{k l i j}^{\varepsilon}\right\rangle, \quad \bar{R}^{\theta}=\left\langle R^{\theta}+R_{i j}^{\varepsilon} A_{i j}^{\theta}\right\rangle .
\end{gathered}
$$

\subsection{Macroscopic problem}

The equations of the macroscopic problem $(8)_{2},(13)_{2},(11),(16)$ are written in the linearized, incremental form as

$$
\begin{gathered}
\delta \bar{\varepsilon}_{i j}=\frac{1}{2}\left[\frac{\partial \delta \bar{u}_{i}}{\partial \bar{x}_{j}}+\frac{\partial \delta \bar{u}_{j}}{\partial \bar{x}_{i}}\right], \quad \delta \bar{\nabla}_{i}=\frac{\partial \delta \bar{\theta}}{\partial \bar{x}_{i}}, \\
\frac{\partial}{\partial \bar{x}_{j}}\left(\bar{\sigma}_{i j}+\delta \bar{\sigma}_{i j}\right)=0, \quad \frac{\partial}{\partial x_{i}}\left(\bar{q}_{i}+\delta \bar{q}_{i}\right)=\bar{r}+\delta \bar{r},
\end{gathered}
$$

where $\bar{r}=\bar{\sigma}_{i j} \dot{\bar{\varepsilon}}_{i j}-\dot{\bar{U}}=\left\langle r^{(0)}\right\rangle$. These equations are accompanied by appropriate, incremental, constitutive relations of the form

$$
\delta \bar{\sigma}_{i j}=\bar{D}_{i j k l}^{\varepsilon} \delta \bar{\varepsilon}_{k l}+\bar{D}_{i j}^{\theta} \delta \bar{\theta}, \quad \delta \bar{r}=\bar{R}_{i j}^{\varepsilon} \delta \bar{\varepsilon}_{i j}+\bar{R}^{\theta} \delta \bar{\theta}
$$

and

$$
\delta \bar{q}_{i}=-\bar{\kappa}_{i j} \delta \bar{\nabla}_{j} .
$$

The parameters $\bar{D}_{i j k l}^{\varepsilon}, \bar{D}_{i j}^{\theta}, \bar{R}_{i j}^{\varepsilon}, \bar{R}^{\theta}$ and $\bar{\kappa}_{i j}$ are provided by the second step of the unit cell problem. The macroscale and microscale analyses are solved simultaneously, according to the iterative framework demonstrated in Figure 1. A proper numerical scheme requires three main algorithms: i) the macroscale algorithm that solves the macroscale problem, ii) the microscale algorithm that solves the unit cell problem and each macroscopic point and iii) the macrothermomechanical tangent moduli that are computed at each unit cell using the solution of the unit cell problem. Analogous algorithms exist in the literature (see for example Terada and Kikuchi, 2001; Tsalis et al., 2013 for elastoplastic composites, or Chatzigeorgiou et al., 2015 for shape memory alloy composites). Here a similar algorithmic scheme with the one proposed in the Appendix A of Chatzigeorgiou et al., 2015 is utilized. The numerical procedure is appropriately extended to account for the microscopic and macroscopic energy equations (15), (16) and the thermal tangent moduli $R_{i j}^{\varepsilon}, R^{\vartheta}$ with their macroscopic counterparts.

REMARK. For positive definite tensors $D_{i j k l}^{\varepsilon}$ and $\kappa_{i j}$ at all times it can be proven that the first terms of equations $(7)_{1,2}$ are always independent of the microscale coordinates (Ene, 1983; Kalamkarov and Kolpakov, 1997; Chatzigeorgiou et al., 2012). 


\section{Discussion about the homogenization framework}

Based on the analysis presented in the previous two sections, the following conclusions can be deduced:

- According to the asymptotic expansion homogenization framework, in the case of smooth media where no displacement or temperature jumps due to cracks are present and no strain or temperature gradient singularities appear due to instability phenomena (i.e. $u_{i}^{\epsilon}, \theta^{\epsilon}, \varepsilon_{i j}^{\epsilon}$ and $\nabla \theta_{i}^{\epsilon}$ are bounded), the displacement vector and the temperature are strongly convergent functions: $u_{i}^{\epsilon}$ and $\theta^{\epsilon}$ coincide asymptotically with their macroscopic counterparts $\bar{u}_{i}$ and $\bar{\theta}$ respectively (see (7) $)_{1,2}$ for $\epsilon \rightarrow 0$ ). The fluctuating parts $u_{i}^{(1)}$ and $\theta^{(1)}$ are influencing only the microscopic strain tensor and the microscopic temperature gradient vector respectively, which can vary significantly inside the unit cell. As a consequence, material constants that may depend on the temperature (for instance the thermal conductivity $\kappa_{i j}$ ) should be considered as functions of the macrotemperature even in the microscale.

- Equivalent results with the approach presented here can be obtained by using the methodology of Suquet (1987). In the zeroth order periodic homogenization, one can alternatively consider that the displacement and the temperature in the microscale are expressed by two terms, a macroscopic and a fluctuating,

$$
u_{i}^{\text {micro }}=\bar{\varepsilon}_{i j} x_{j}+\tilde{u}_{i}, \quad \theta^{\text {micro }}=\bar{\nabla}_{i} x_{i}+\tilde{\theta} .
$$

Taking the spatial derivatives of these expressions, the strain and the temperature gradient are given by relations analogous to those of (8) and (13) respectively. Even though equations (51) are very efficient in expressing the unit cell problem, they cannot be used to determine the macroscopic displacement and temperature, since $\tilde{u}_{i}$ and $\tilde{\theta}$ depend not only on the microscopic position, but also on the size of the unit cell (i.e. the characteristic length $\epsilon$ ). Thus, one needs to postulate the constitutive law of a thermomechanical medium as function of the macrotemperature (see Maghous and Creus, 2003). The same hypothesis is also considered in the case of micromechanics of general composite media (Rosen and Hashin, 1970). The advantage of the current formulation is that such postulation is not required, since it arises naturally from the expanded form $(7)_{2}$ of the temperature.

- The developed framework reveals that a fully coupled thermomechanical analysis for periodic composites through homogenization has two significant differences compared to a corresponding analysis for homogeneous materials:

i) The microequilibrium (10) is solved under isothermal macroscopic temperature, while equation (15) states that the balance of energy in the microscale includes only the heat conduction part. Thus, the microscale equilibrium and energy equation are practically uncoupled and they are solved separately, independently of how complicated the expressions of the micro-energy potentials are. The coupling arising from the thermomechanical energetic terms and the mechanical dissipation is taken into account on the macroscale level through the energy 
equation (16). This result contradicts with the hypothesis of Sengupta et al. (2012), where a displacement-like thermal boundary condition is imposed in both microscopic conservation laws.

ii) The linearized incremental formulation yields that the macroscopic tangent moduli related with the energy equation $\left(\bar{R}_{i j}^{\varepsilon}\right.$ and $\left.\bar{R}^{\theta}\right)$ are provided by the linearized equations $(47)_{4,5}$. To the best of the authors knowledge, these two equations are introduced for the first time in the homogenization theories for small deformation processes (for both periodic and random media). They actually correlate the macroscopic tangent moduli $\bar{R}_{i j}^{\varepsilon}$ and $\bar{R}^{\theta}$ with a) their microscopic counterparts (which are not involved in the solution of the microscale problem since it is decoupled) and b) the concentration tensors obtained exclusively from the solution of the microequations $(44)_{1,2}$. This novel formalism of the current work could perhaps be useful in the case of random media, in which the Eshelby-based approaches provide analytical or semi-analytical expressions for the concentration tensors $A_{i j k l}^{\varepsilon}$ and $A_{i j}^{\theta}$ (Benveniste et al., 1991).

- Combining equation (38) with $(30)_{1,3}$ or $(30)_{2,4}$ yields

$$
r^{(0)}=-\bar{\theta} \dot{S}^{(0)}+\gamma_{\mathrm{loc}}^{(0)}
$$

In this representation, the $r^{(0)}$ can be considered as the sum of the local dissipation and the power arising from the entropy rate. In the linearized, incremental formulation, one can identify relations for the entropy and the dissipation increments of the form

$$
\delta S^{(0)}=S_{i j}^{\varepsilon} \delta \varepsilon_{i j}^{(0)}+S^{\theta} \delta \bar{\theta}, \quad \delta \gamma_{\mathrm{loc}}^{(0)}=\Gamma_{i j}^{\varepsilon} \delta \varepsilon_{i j}^{(0)}+\Gamma^{\theta} \delta \bar{\theta}
$$

The first expression of (53) provides a constitutive relation between the entropy, the temperature and the strain. Such type of constitutive law can be very useful (in extended form) in the case of multiphysics homogenization problems with additional fields like magnetic or electric (see for instance Bravo-Castillero et al., 2009). The second expression of (53) is considered as a linearized representation of the local dissipation. Combining (52) and (53) gives

$$
\delta r^{(0)}=\left[-\frac{\bar{\theta}}{\Delta t} S_{i j}^{\varepsilon}+\Gamma_{i j}^{\varepsilon}\right] \delta \varepsilon_{i j}^{(0)}+\left[-\frac{\Delta s^{(0)}}{\Delta t}-\frac{\bar{\theta}}{\Delta t} S^{\theta}+\Gamma^{\theta}\right] \delta \bar{\theta} .
$$

With the help of the last expression and equations (45) and (46), the macroscopic thermal tangent moduli $\bar{R}_{i j}^{\varepsilon}$ and $\bar{R}^{\theta}$ can be obtained.

\section{Numerical examples}

The examples presented in this section illustrate the response of a viscoplastic multilayered composite under thermomechanical loading conditions. In order to demonstrate the framework capabilities, the material constituents are considered to have high contrast in material properties. Figure 2 shows the composite's microstructure. Two materials (an epoxy, with volume fraction vf, and a metal, with volume fraction 1-vf) are bonded, forming a multilayered 


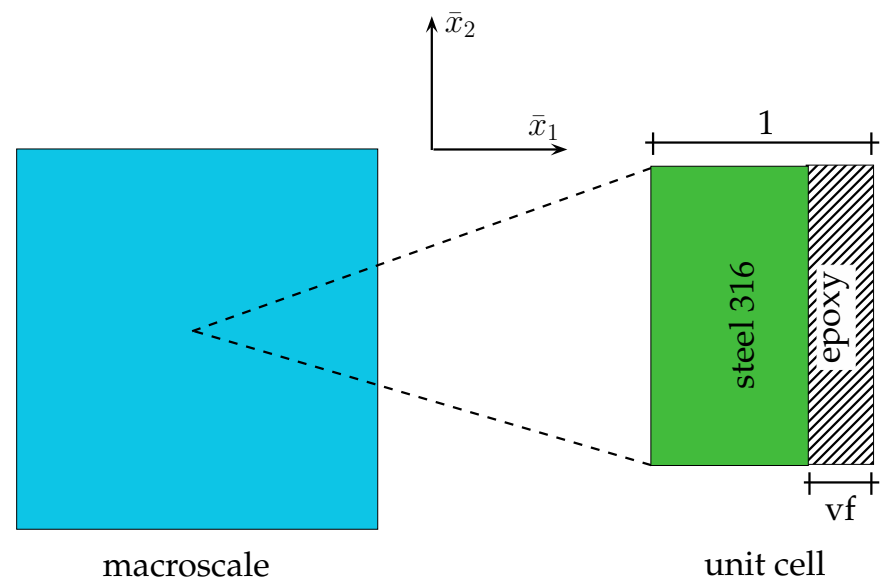

Figure 2: Multilayered composite: Each macroscopic point is represented by a unit cell consisting of two material layers, an epoxy and a metal.

structure which is repeated periodically in the overall composite. The epoxy is assumed to behave elastically, while the steel 316 is considered viscoplastic material. When plastically deformed, the von Mises stress $\sigma^{\mathrm{vM}}$ is expressed in terms of the accumulated plastic strain $p$ and its rate according to the relation (Lemaitre and Chaboche, 2002)

$$
\sigma^{\mathrm{vM}}=Y+Q_{1} p+Q_{2}[1-\exp (-b p)]+K_{a} \dot{p}^{1 / N_{a}}
$$

where $Y$ is the elastic limit, $Q_{1}, Q_{2}, b$ are plastic hardening parameters and $K_{a}, N_{a}$ are viscous parameters. The material parameters of both the metal and the epoxy are shown in Table 3.

Table 3: Thermomechanical properties of constituents. The steel viscoplastic properties are provided in Lemaitre and Chaboche (2002).

\begin{tabular}{lrr} 
property & steel 316 & epoxy \\
\hline Young modulus [MPa] & 185000 & 3000 \\
Poisson ratio & 0.27 & 0.3 \\
thermal expansion $[1 / \mathrm{K}]$ & $1.5 \mathrm{E}-5$ & $1.1 \mathrm{E}-4$ \\
density $\left[\mathrm{t} / \mathrm{m}^{3}\right]$ & 7.8 & 1.12 \\
specific heat capacity $[\mathrm{MJ} /(\mathrm{t} \mathrm{K})]$ & 0.5 & 1.64 \\
thermal conductivity $[\mathrm{MN} /(\mathrm{s} \mathrm{K})]$ & $1.5 \mathrm{E}-5$ & $0.4 \mathrm{E}-6$ \\
elastic limit $[\mathrm{MPa}]$ & 84 & \\
$K_{a}[\mathrm{MPa}]$ & 151 & \\
$N_{a}$ & 24 & \\
$Q_{1}[\mathrm{MPa}]$ & 6400 & \\
$Q_{2}[\mathrm{MPa}]$ & 270 & \\
$b$ & 25 &
\end{tabular}




\subsection{Computation of the macroscopic response of the composite material}

The macroscopic response of the composite is computed using a specifically developed themomechanical solver in Fortan. Indeed, the advantage of the multilayered composite is that the unit cell problem has a semi-analytical solution (see for instance Tsalis et al., 2013; Chatzigeorgiou et al., 2015 for elastoplastic and shape memory alloy composites respectively). While in typical composites like fiber, particulate, woven, e.t.c. the unit cell problem requires a FE analysis, leading to the so-called " $\mathrm{FE}^{2}$ " method when solving simultaneously the micro and macro equations, the multilayered composite can avoid the finite element calculations in the microscale since it is actually a 1-D problem. Such simplification reduces drastically the computational cost. The constitutive laws implementation for the elastic epoxy and the viscoplastic metal are described in Appendix A. In the subsequent analyses the reference temperature $\theta_{0}$ is taken equal to $293.15^{\circ} \mathrm{K}\left(20^{\circ} \mathrm{C}\right)$.

\subsubsection{Isothermal conditions}

The fist numerical tests are performed under isothermal conditions $\left(20^{\circ} \mathrm{C}\right)$ and uniaxial stress loading in the direction $\bar{x}_{1}$, normal to the layers (Figure 3). Four different epoxy volume fractions are chosen $(0 \%, 20 \%, 40 \%$ and $60 \%)$. The

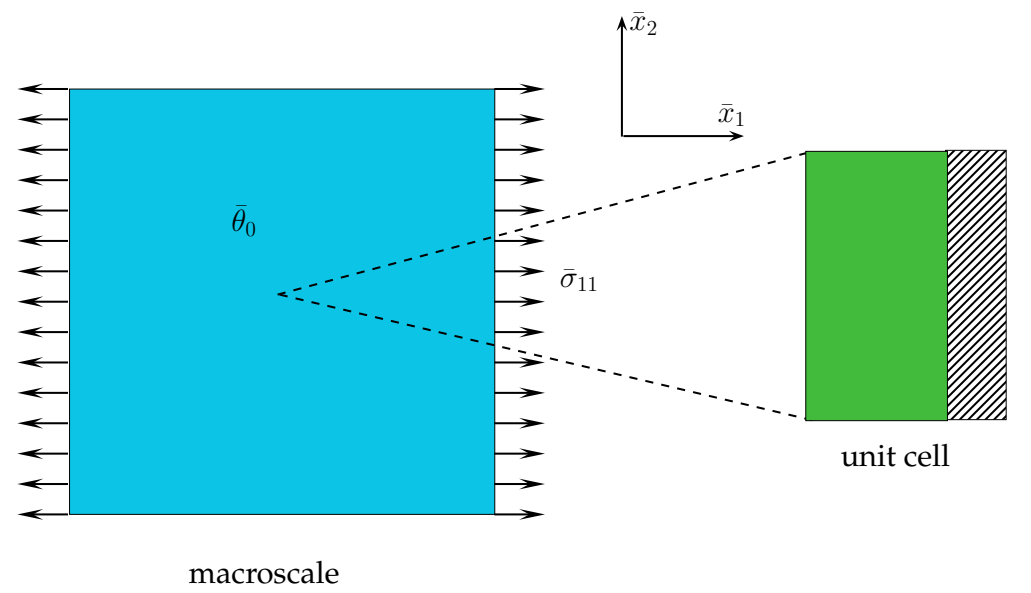

Figure 3: Multilayered composite: Uniaxial response under isothermal conditions.

uniaxial stress loading is conducted under stress rate $1 \mathrm{MPa} / \mathrm{s}$ and includes the following steps:

1. loading up to $200 \mathrm{MPa}$ and subsequent unloading,

2. loading up to $220 \mathrm{MPa}$ and subsequent unloading,

3. loading up to $240 \mathrm{MPa}$ and subsequent unloading.

Figure 4 presents the obtained macroscopic stress-strain curves and the evolution of the macroscopic local dissipation with time. As expected, the increase of the epoxy volume fraction decreases drastically the composite's Young modulus. On the other hand, the decrease of the dissipative material's volume fraction causes a reduction on the composite's dissipation energy rate per loading cycle. 


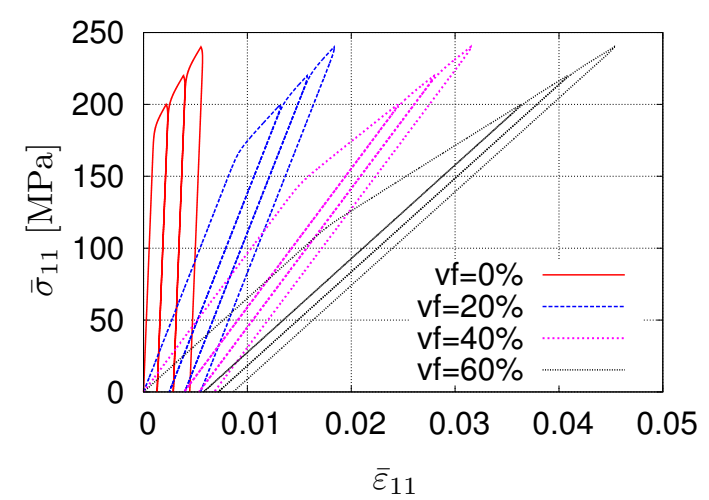

(a)

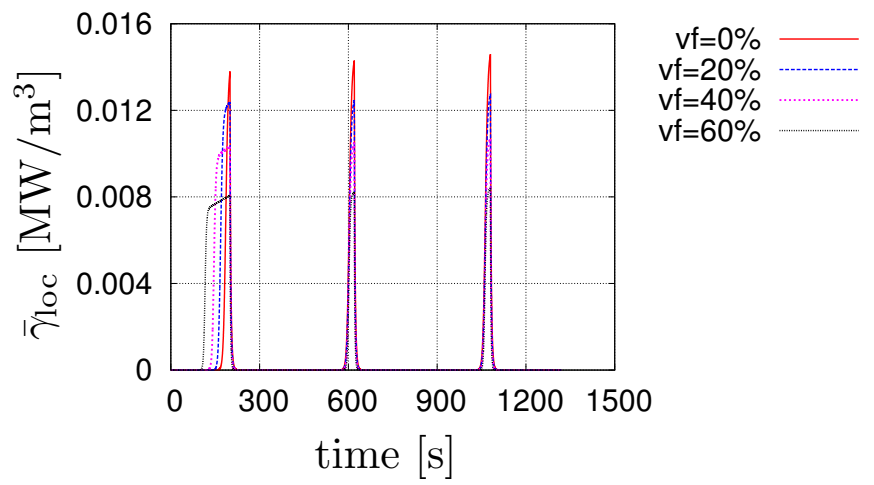

(b)

Figure 4: Uniaxial loading (1 MPa/s) of multilayered composite under isothermal conditions: (a) macroscopic stress-strain curve and (b) evolution of macroscopic local dissipation with time for four epoxy volume fractions.

\subsubsection{Adiabatic conditions}

The second series of numerical tests are considered under adiabatic conditions and uniaxial stress loading in the direction $\bar{x}_{1}$, normal to the layers. (Figure 5). The initial macroscopic temperature is assumed $20^{\circ} \mathrm{C}$. Two different

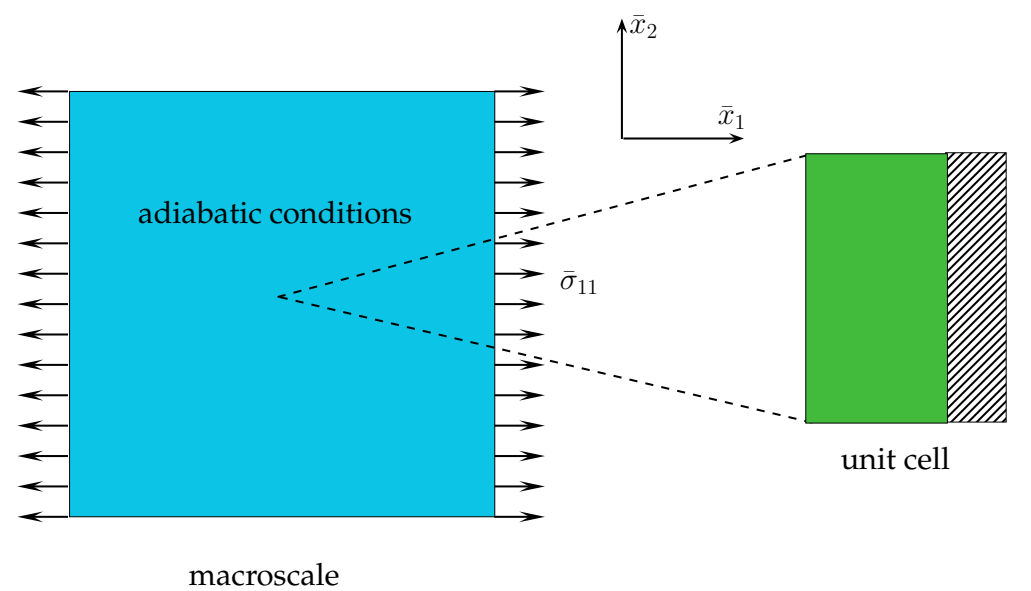

Figure 5: Multilayered composite: Uniaxial response under adiabatic conditions.

epoxy volume fractions are chosen $(0 \%$ and $20 \%)$. The uniaxial stress loading is conducted under stress rate $1 \mathrm{MPa} / \mathrm{s}$ and the steps are similar to those of the previous subsection. Figure 6 shows the obtained macroscopic stress-strain curves and the evolution of the macroscopic temperature with time. The stress-strain responses remain similar to the corresponding of the isothermal case. Moreover, the composite with $20 \%$ epoxy shows higher variations in the temperature during a cycle (maximum $1^{\circ} \mathrm{C}$ ) compared to the pure metal (maximum $0.3^{\circ} \mathrm{C}$ ). 


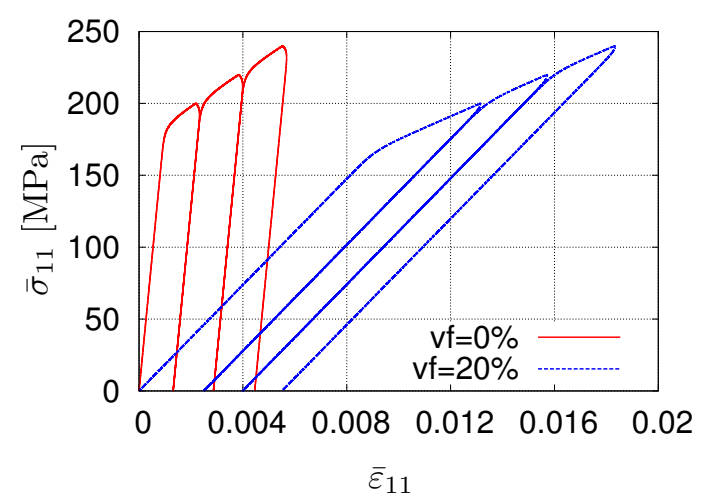

(a)

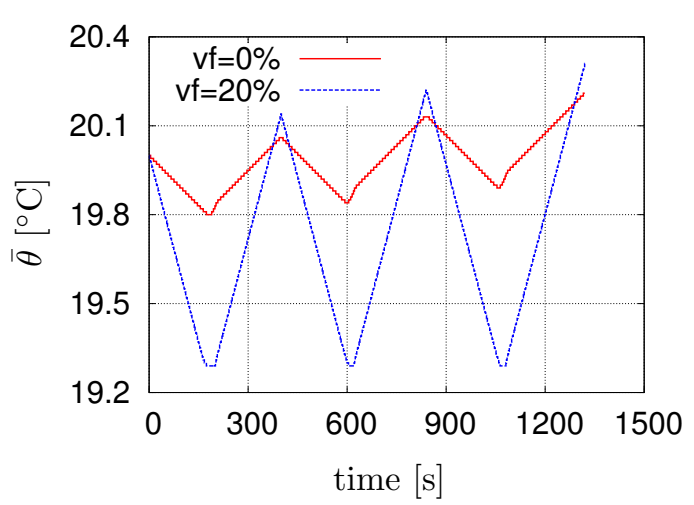

(b)

Figure 6: Uniaxial loading (1 MPa/s) of multilayered composite under adiabatic conditions: (a) macroscopic stress-strain curve and (b) evolution of temperature with time for two epoxy volume fractions.

\subsection{Simulation of the behavior of a composite structure}

The global thermomechanical response of a composite structure can be computed using finite element analysis (FEA), which has to be performed only at the structural level, using the semi-analytical solution for multilayered composites. In what follows, it is supposed that integration points of the FEA model are considered to be material points that are representative of the composite microstructure (i.e. one material point contains a significant amount of unit cells). The necessary calculations to obtain the evolution of internal variables for the constitutive model of the phases, along with the homogenization scale transition scheme, are computed at each integration point through a specific subroutine.

Specifically, the solution of the mechanical part of the unit cell problem (equations (37) 1 and (37) 3 , accompanied by constitutive laws for each material phase in the microscale), as well as the solution of the equations $(44)_{1,2}$ and the computation of the macroscopic tangent moduli $(47)_{1,2,4,5}$ are introduced in a specially designed User Material subroutine (UMAT) for the commercial finite element software ABAQUS. The thermal equations $(37)_{2}$ and (37) 4 are not required to be solved explicitly. Due to the linearity of the thermal problem, the macroscopic thermal conductivity $(47)_{3}$ and the thermal concentration tensors $(46)_{3}$ are computed analytically for a multilayered composite, allowing to obtain the necessary information for the macroscopic energy equation and to determine the temperature gradients and heat fluxes vectors of each layer in the microstructure. The designed UMAT serves as a "constitutive law" for the macroscale problem, which is solved numerically with the help of ABAQUS.

Numerical calculations on cubic specimen with dimensions $1 \mathrm{~cm} \times 1 \mathrm{~cm} \times 1 \mathrm{~cm}$ are performed with the help of the commercial software ABAQUS. 1000 C3D8T elements (8 node, 3-D solid elements for coupled temperaturedisplacement analysis) are utilized to represent the macrostructure, in which the constitutive law is given by the solution of the unit cell problem. The microstructure of this composite is considered to consist of the two layers of Figure 2, where the $\bar{x}_{1}$ direction corresponds to the $\mathrm{X}$ direction in the FE analysis (Figures $7_{a}$ and 8 ). The epoxy volume fraction is taken equal to $20 \%$. From the solution of the thermal microequations $(37)_{2}$ and $(37)_{4}$ for a multilayered composite it is found that the macroscopic thermal conductivity (equation $(47)_{3}$ ) presents transverse isotropy 
with the $\bar{x}_{1}$ as the axis of symmetry. The $\bar{\kappa}_{11}$ is defined as the inverse rule of mixtures and is equal to $1.807 \mathrm{E}-06$, while the $\bar{\kappa}_{22}$ and $\bar{\kappa}_{33}$ are defined as the rule of mixtures and are equal to $1.208 \mathrm{E}-05$ (see for instance Chatzigeorgiou et al., 2012).

The macrostructure is subjected to the following conditions (Figures $7_{b}$ and 8):

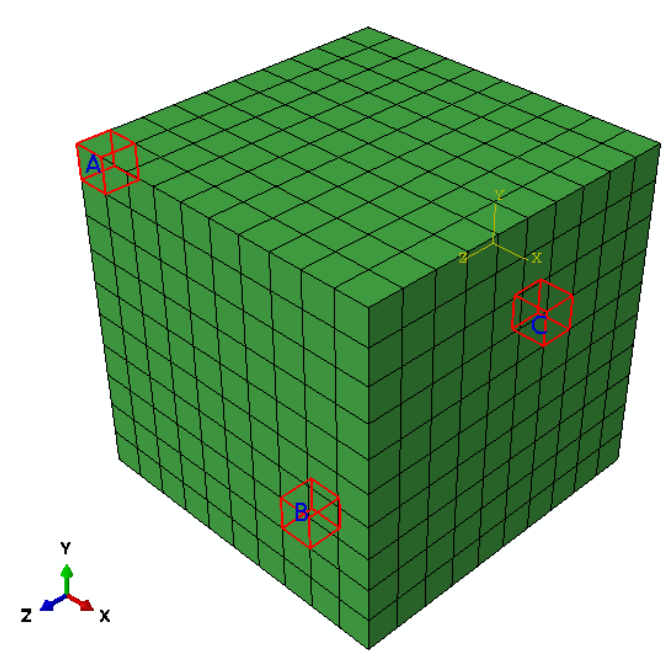

(a)

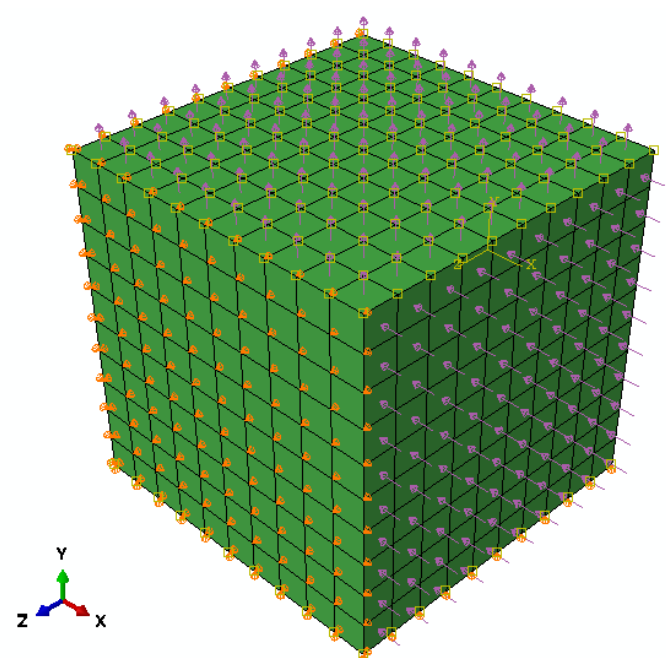

(b)

Figure 7: (a) Finite element meshing of macrostructure. (b) Boundary and loading conditions on the meshed macrostructure.

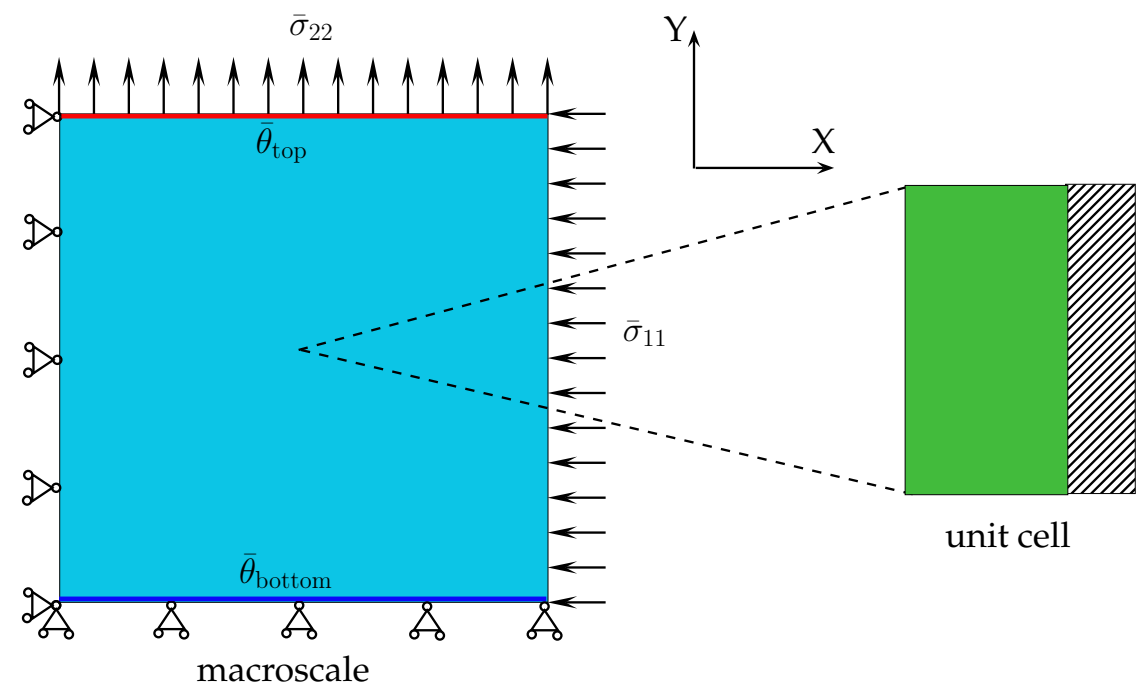

Figure 8: Two dimensional representation of the boundary conditions and thermomechanical loading configuration (biaxial loading and temperature difference).

- initial temperature of the structure $293.15^{\circ} \mathrm{K}\left(20^{\circ} \mathrm{C}\right)$, 
- fixed displacement in the Y direction of the bottom surface,

- fixed displacement in the $\mathrm{Z}$ direction of the front surface,

- fixed displacement in the $\mathrm{X}$ direction of the left surface,

- linearly increasing tensile stress $\bar{\sigma}_{22}$ with time, up to $150 \mathrm{MPa}$, in the upper surface (normal to Y direction),

- linearly increasing compressive stress $\bar{\sigma}_{11}$ with time, up to $150 \mathrm{MPa}$, in the right surface (normal to X direction),

- linearly increasing temperature $\bar{\theta}_{\text {top }}$ with time, up to $303.15^{\circ} \mathrm{K}\left(30^{\circ} \mathrm{C}\right)$, in the upper surface,

- fixed temperature $\bar{\theta}_{\text {bottom }}$ with time at $293.15^{\circ} \mathrm{K}\left(20^{\circ} \mathrm{C}\right)$ in the bottom surface.

In order to demonstrate the rate-dependency of the composite, two cases are considered: a) total time of the analysis $200 \mathrm{~s}$ and b) total time of the analysis $20000 \mathrm{~s}$. Certain results are generated at the centroids of the elements A, B and C shown in Figure $7 a$.

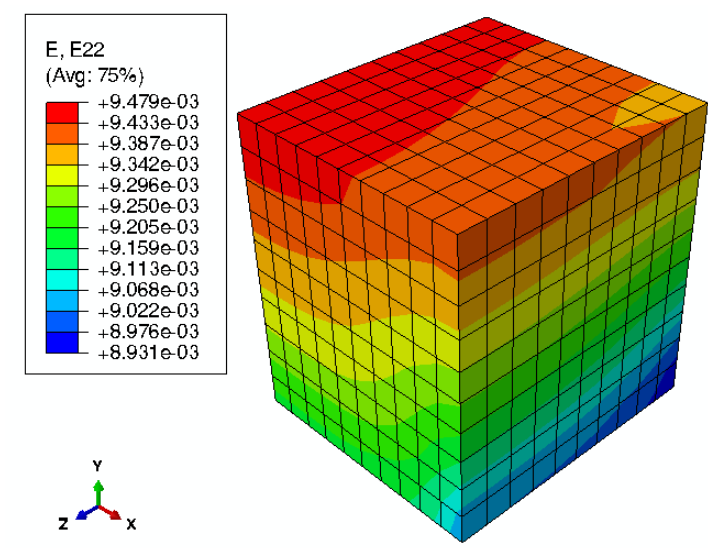

(a)

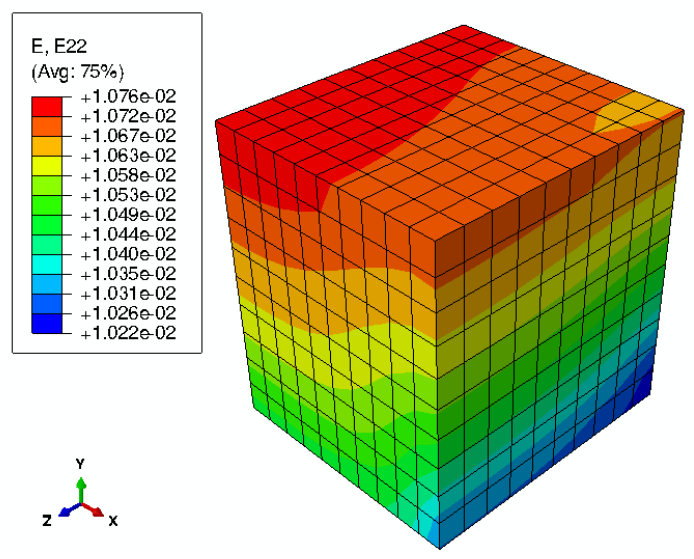

(b)

Figure 9: Strain $\bar{\varepsilon}_{22}$ distribution in the macrostructure for total analysis time of a) $200 \mathrm{~s}$ and b) $20000 \mathrm{~s}$. Composite with $20 \%$ epoxy volume fraction.

Figure 9 shows the normal strain $\bar{\varepsilon}_{22}$ distribution at the end of the thermomechanical loading path. As it is expected, longer exposure of the specimen in high stresses causes increase in the developed strains due to the viscoplastic behavior of the steel 316 component. On the other hand, the temperature is practically insensitive to the loading rate (Figure 10) and has an almost linear spatial variation.

The microscopic as well as the macroscopic stresses in the direction Y, developed at the centroid of the macro-element $\mathrm{A}$, are shown as a function of the macrostrain $\bar{\varepsilon}_{22}$ in Figure 11. It is observed that the epoxy is under compression, with stress value which is almost stabilized once the steel 316 enters into the plastic regime. 


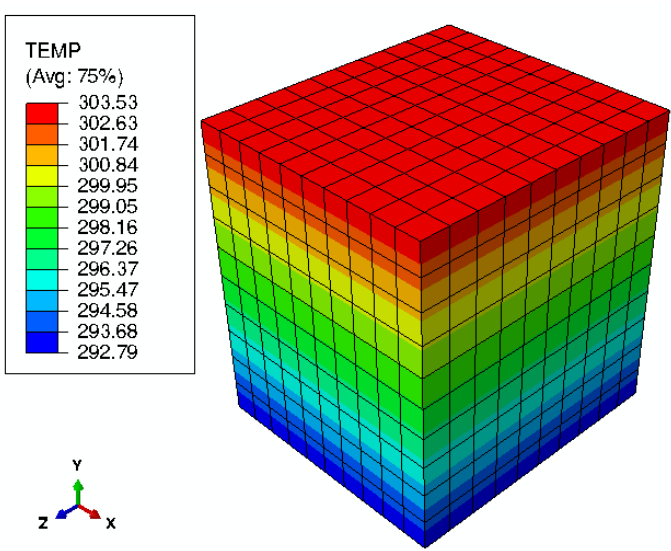

(a)

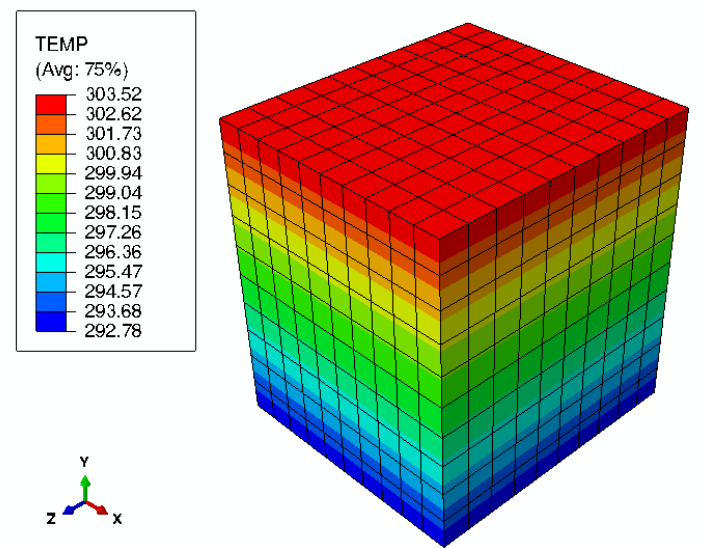

(b)

Figure 10: Temperature distribution (in K) in the macrostructure for total analysis time of a) $200 \mathrm{~s}$ and b) $20000 \mathrm{~s}$. Composite with $20 \%$ epoxy volume fraction.

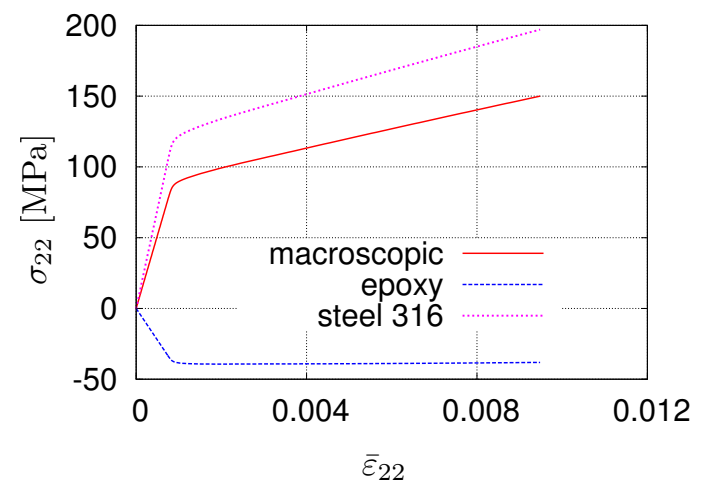

(a)

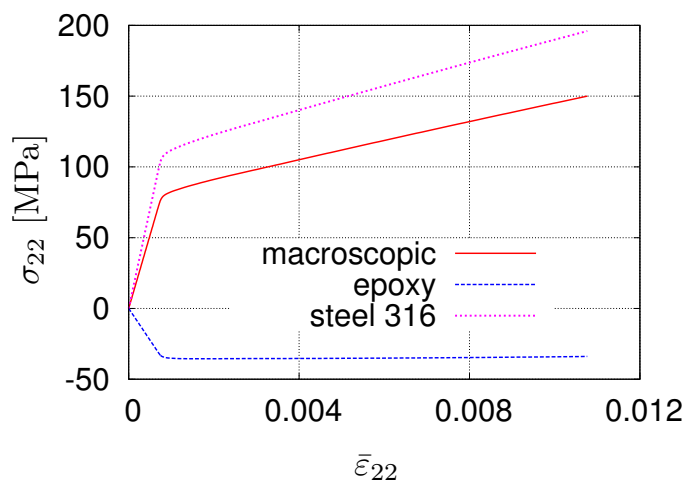

(b)

Figure 11: Evolution of macroscopic and microscopic stresses with strain at the centroid of the element A (Figure $7_{a}$ ) for total analysis time of a) $200 \mathrm{~s}$ and b) $20000 \mathrm{~s}$. Composite with $20 \%$ epoxy volume fraction.

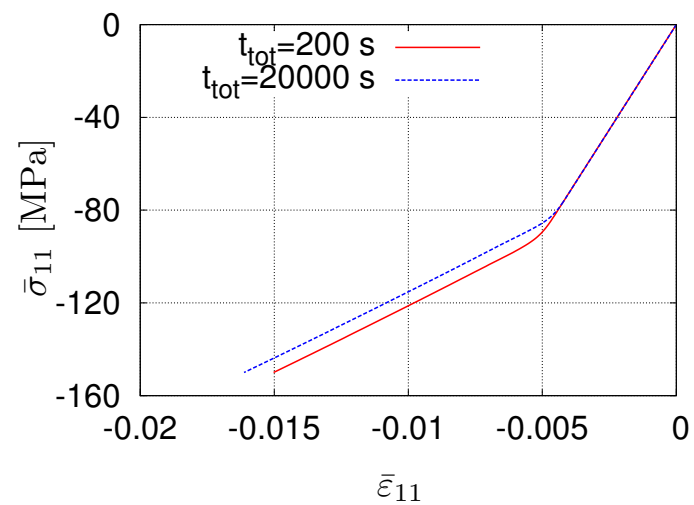

Figure 12: Macroscopic stress-strain in the direction 1 at the centroid of the element B (Figure $7_{a}$ ) for two different loading durations. Composite with $20 \%$ epoxy volume fraction. 
As it is shown in Figure 12, the stress-strain response at the plastic regime of the composite depends on the loading rate. Specifically, increase of the test duration by 100 times causes an increase of $0.1 \%$ in the strain level in direction $\mathrm{Y}$ of the macro-element B.

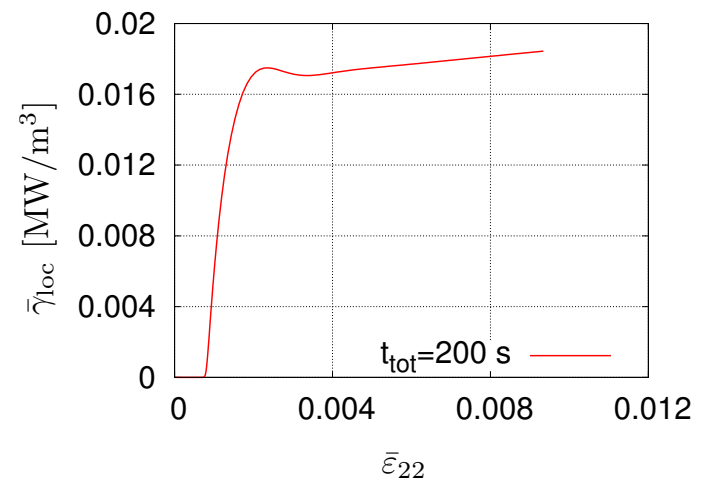

(a)

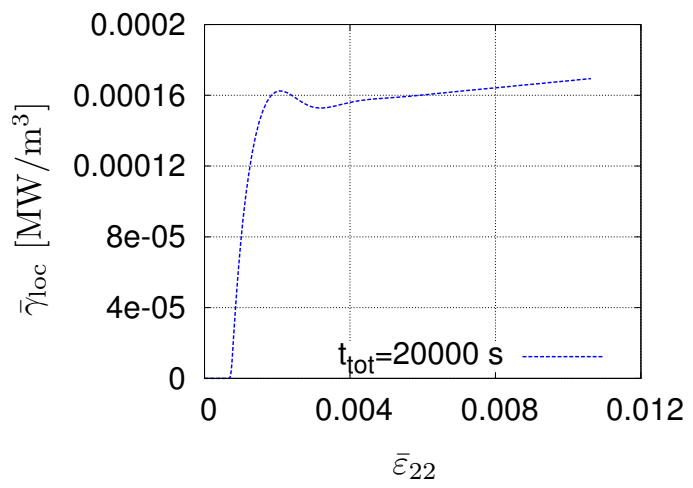

(b)

Figure 13: Macroscopic local dissipation distribution at the centroid of the element $\mathrm{C}$ (Figure $7_{a}$ ) for total analysis time of a) $200 \mathrm{~s}$ and b) $20000 \mathrm{~s}$. Composite with $20 \%$ epoxy volume fraction.

Finally, Figure 13 illustrates the evolution of the macroscopic local dissipation in the centroid of macro-element $\mathrm{C}$ for loading durations $200 \mathrm{~s}$ and $20000 \mathrm{~s}$. As observed, the local dissipation has a high increase when the composite enters in the plastic regime, followed by a short small decrease and a long, continuous slight increase.

\section{Conclusion remarks}

The periodic homogenization framework developed in this manuscript is capable of describing the fully coupled thermomechanical response of a composite consisting of materials with nonlinear, dissipative response. Using the principles of the asymptotic expansion homogenization method the main equations (equilibrium, thermodynamics laws, constitutive relations) are described in both macro and microlevels. The difficulty of having an analytical expression for the macroscopic energy equation in terms of "macroscopic" internal variables is bypassed by introducing a linearized incremental formulation that expresses the necessary terms as functions of the total strain and temperature. Numerical examples on multilayered composites with viscoplastic constituents illustrated the capabilities of the proposed methodology to identify both the microscopic and macroscopic fields under various thermomechanical conditions.

From a practical point of view, the new framework can be used in the cases of composites where the interplay between mechanical and thermal fields is strong and fully coupled thermomechanical analysis is required. Polymeric materials are characterized by viscoelastic or viscoplastic behavior and their mechanical properties are very sensitive to temperature variations. Thus, for polymeric composites under fatigue tests, the mechanical dissipation produced by the viscous mechanisms causes increase in the material's temperature, which in turn affects the mechanical properties 
of the polymeric matrix. In such occasions the designed framework could be very useful to account properly such complicated composite responses.

\section{Appendix A. Incremental, linearized forms of tangent moduli for various materials}

The derivation of appropriate tangent moduli of the material constituents is essential for the solution of the system of thermomechanical equations in a linearized, incremental framework. In order to establish these moduli, it is necessary to connect them with a suitable energy potential. For a Gibbs free energy of the form $G\left(\sigma_{i j}, \theta, \zeta_{\alpha}\right)$, it can be shown, using equations $(24)_{2},(25)_{3,4},(26)_{1,3}$ and (28), that the following relations for generalized standard materials hold:

$$
\varepsilon_{i j}=-\frac{\partial G}{\partial \sigma_{i j}}, \quad s=-\frac{\partial G}{\partial \theta}, \quad \gamma_{\mathrm{loc}}=-\frac{\partial G}{\partial \zeta_{\alpha}} \dot{\zeta}_{\alpha}, \quad r=\sigma_{i j} \dot{\varepsilon}_{i j}-\dot{U}=-\theta \dot{s}+\gamma_{\mathrm{loc}} .
$$

According to the type of material that is considered, these expressions obtain specific form (Lemaitre and Chaboche, 2002). Here, four types of generalized standard materials are examined.

\section{Thermoelastic material}

Consider a classical thermoelastic material initially at a state of reference temperature $\theta_{0}$. The Gibbs free energy potential in this case is written as ${ }^{2}$

$$
G\left(\sigma_{i j}, \theta\right)=-\frac{1}{2} \sigma_{i j} C_{i j k l}^{-1} \sigma_{k l}-\alpha_{i j} \sigma_{i j}\left[\theta-\theta_{0}\right]+c_{0}\left[\left[\theta-\theta_{0}\right]-\theta \ln \left(\frac{\theta}{\theta_{0}}\right)\right]-s_{0} \theta+U_{0}
$$

where $C_{i j k l}, \alpha_{i j}, c_{0}, s_{0}$ and $U_{0}$ are material constants. Moreover, $C_{i j k l}$ is a fourth order tensor with major and minor symmetries and $\alpha_{i j}$ is a symmetric second order tensor. Since no mechanical dissipation occurs in a thermoelastic material, $\gamma_{\mathrm{loc}}=0$. Using (A.1) yields

$$
\sigma_{i j}=C_{i j k l}\left[\varepsilon_{k l}-\alpha_{k l}\left[\theta-\theta_{0}\right]\right], \quad s=\alpha_{i j} \sigma_{i j}+c_{0} \ln \left(\frac{\theta}{\theta_{0}}\right)+s_{0}, \quad r=-c_{0} \dot{\theta}-\theta \alpha_{i j} \dot{\sigma}_{i j}
$$

Expressing the above relation in the incremental, linearized form described in subsection 3.1, the following incremental formulations are obtained:

$$
\begin{gathered}
\delta \sigma_{i j}=C_{i j k l} \delta \varepsilon_{k l}-C_{i j k l} \alpha_{k l} \delta \theta, \\
\delta r=-\frac{c_{0}}{\Delta t} \delta \theta-\frac{\theta}{\Delta t} \alpha_{i j} \delta \sigma_{i j}-\alpha_{i j} \frac{\Delta \sigma_{i j}}{\Delta t} \delta \theta=-\frac{\theta}{\Delta t} \alpha_{i j} C_{i j k l} \delta \varepsilon_{k l}-\frac{1}{\Delta t}\left[c_{0}+\alpha_{i j} \Delta \sigma_{i j}-\theta \alpha_{i j} C_{i j k l} \alpha_{k l}\right] \delta \theta .
\end{gathered}
$$

From the above relations it is clear that

$$
D_{i j k l}^{\varepsilon}=C_{i j k l}, \quad D_{i j}^{\theta}=-C_{i j k l} \alpha_{k l}, \quad R_{i j}^{\varepsilon}=-\frac{\theta}{\Delta t} \alpha_{k l} D_{k l i j}^{\varepsilon}, \quad R^{\theta}=-\frac{1}{\Delta t}\left[c_{0}+\alpha_{i j} \Delta \sigma_{i j}+\theta \alpha_{i j} D_{i j}^{\theta}\right]
$$

\footnotetext{
${ }^{2}$ The inverse of a fourth order symmetric tensor $C_{i j k l}$ is defined as the fourth order symmetric tensor $C_{i j k l}^{-1}$ for which it holds $C_{i j m n} C_{m n k l}^{-1}=$ $\frac{1}{2} \delta_{i k} \delta_{j l}+\frac{1}{2} \delta_{i l} \delta_{j k}$, where $\delta_{i j}$ is the Kronecker delta.
} 


\section{$J_{2}$ thermoelastoplastic material with isotropic hardening}

The thermodynamic potential of a $\mathbf{J}_{2}$ thermoelastoplastic material with isotropic hardening depends on internal variables related with the plastic mechanism. A typical form of such potential is

$$
G\left(\sigma_{i j}, \theta, \varepsilon_{i j}^{p}, p\right)=-\frac{1}{2} \sigma_{i j} C_{i j k l}^{-1} \sigma_{k l}-\alpha_{i j} \sigma_{i j}\left[\theta-\theta_{0}\right]-\sigma_{i j} \varepsilon_{i j}^{p}+c_{0}\left[\left[\theta-\theta_{0}\right]-\theta \ln \left(\frac{\theta}{\theta_{0}}\right)\right]-s_{0} \theta+U_{0}+f(p)
$$

In the above expression $\varepsilon_{i j}^{p}$ denotes the second order plastic strain tensor, $p$ is the accumulated plastic strain scalar and $f(p)$ is a hardening related function. Moreover, $C_{i j k l}, \alpha_{i j}, c_{0}, s_{0}$ and $U_{0}$ are material constants similar to the ones of a thermoelastic material. Using (A.1) and defining $F:=F(p)=\frac{\mathrm{d} f}{\mathrm{~d} p}$ yields

$$
\sigma_{i j}=C_{i j k l}\left[\varepsilon_{k l}-\varepsilon_{k l}^{p}-\alpha_{k l}\left[\theta-\theta_{0}\right]\right], \quad \gamma_{\mathrm{loc}}=\sigma_{i j} \dot{\varepsilon}_{i j}^{p}-F \dot{p}, \quad r=-c_{0} \dot{\theta}-\theta \alpha_{i j} \dot{\sigma}_{i j}+\gamma_{\mathrm{loc}}
$$

In generalized standard materials the local dissipation $\gamma_{\text {loc }}$ can be expressed in terms of a dissipation potential (Lemaitre and Chaboche, 2002). For a yield surface of the form

$$
\Phi:=\Phi\left(\sigma_{i j}, F\right)=\sigma^{\mathrm{vM}}-F-Y \leq 0, \quad \dot{p} \geq 0, \quad \Phi \dot{p}=0
$$

the dissipation potential provides the evolution equation of the plastic strain

$$
\dot{\varepsilon}_{i j}^{p}=\Lambda_{i j} \dot{p}, \quad \Lambda_{i j}=\frac{3 \sigma_{i j}^{\prime}}{2 \sigma^{\mathrm{vM}}}=\frac{\partial \Phi}{\partial \sigma_{i j}}
$$

In the above expressions

$$
\sigma^{\mathrm{vM}}=\sqrt{\frac{3}{2} \sigma_{i j}^{\prime} \sigma_{i j}^{\prime}}, \quad \sigma_{i j}^{\prime}=\sigma_{i j}-\frac{1}{3} \sigma_{k k} \delta_{i j},
$$

$Y$ is the elastic limit (positive constant) and $\delta_{i j}$ denotes the Kronecker delta. Expressing the above relation in the incremental, linearized form described in subsection 3.1 and utilizing the assumptions of the convex cutting plane technique (Simo and Hughes, 1998; Qidwai and Lagoudas, 2000; Hartl et al., 2010), the following incremental formulations are obtained:

- When plastic strains do not evolve, i.e. $\Phi<0$, the tangent moduli are the same with those of a thermoelastic material (equations (A.5)).

- When plastic strains are generated, i.e. $\Phi=0$, then (Simo and Hughes, 1998)

$$
\begin{aligned}
\delta p & =\frac{1}{\mathcal{A}} \Lambda_{i j} C_{i j k l} \delta \varepsilon_{k l}-\frac{1}{\mathcal{A}} \Lambda_{i j} C_{i j k l} \alpha_{k l} \delta \theta, \quad \mathcal{A}=\Lambda_{i j} C_{i j k l} \Lambda_{k l}+\frac{\mathrm{d} F}{\mathrm{~d} p}, \\
\delta \varepsilon_{i j}^{p} & =\Lambda_{i j} \delta p=\frac{1}{\mathcal{A}} \Lambda_{i j} \Lambda_{p q} C_{p q k l} \delta \varepsilon_{k l}-\frac{1}{\mathcal{A}} \Lambda_{i j} \Lambda_{p q} C_{p q k l} \alpha_{k l} \delta \theta, \\
\delta \sigma_{i j} & =\left[C_{i j k l}-\frac{1}{\mathcal{A}} C_{i j m n} \Lambda_{m n} \Lambda_{p q} C_{p q k l}\right] \delta \varepsilon_{k l}-\left[C_{i j k l}-\frac{1}{\mathcal{A}} C_{i j m n} \Lambda_{m n} \Lambda_{p q} C_{p q k l}\right] \alpha_{k l} \delta \theta .
\end{aligned}
$$

From the above relations it is clear that

$$
D_{i j k l}^{\varepsilon}=C_{i j k l}-\frac{1}{\mathcal{A}} C_{i j m n} \Lambda_{m n} \Lambda_{p q} C_{p q k l}, \quad D_{i j}^{\theta}=-D_{i j k l}^{\varepsilon} \alpha_{k l}
$$


Moreover, the increment of local dissipation (A.7) $)_{2}$, with the help of the identity $\sigma_{i j} \Lambda_{i j}=\sigma^{\mathrm{vM}}$, is written

$$
\delta \gamma_{\mathrm{loc}}=\frac{\Delta \varepsilon_{i j}^{p}}{\Delta t} \delta \sigma_{i j}+\sigma_{i j} \frac{\delta \varepsilon_{i j}^{p}}{\Delta t}-\frac{\Delta p}{\Delta t} \delta F-F \frac{\delta p}{\Delta t}=\Gamma_{i j}^{\varepsilon} \delta \varepsilon_{i j}+\Gamma^{\theta} \delta \theta
$$

where

$$
\begin{aligned}
& \Gamma_{i j}^{\varepsilon}=\frac{\Delta \varepsilon_{k l}^{p}}{\Delta t} D_{k l i j}^{\varepsilon}+\frac{1}{\mathcal{A} \Delta t}\left[\sigma^{\mathrm{vM}}-\Delta p \frac{\mathrm{d} F}{\mathrm{~d} p}-F\right] \Lambda_{k l} C_{k l i j}, \\
& \Gamma^{\theta}=\frac{\Delta \varepsilon_{i j}^{p}}{\Delta t} D_{i j}^{\theta}-\frac{1}{\mathcal{A} \Delta t}\left[\sigma^{\mathrm{vM}}-\Delta p \frac{\mathrm{d} F}{\mathrm{~d} p}-F\right] \Lambda_{i j} C_{i j k l} \alpha_{k l} .
\end{aligned}
$$

Finally, linearization of equation $(\mathrm{A} .7)_{3}$ gives

$$
\delta r=-\frac{\theta}{\Delta t} \alpha_{i j} C_{i j k l}\left[\delta \varepsilon_{k l}-\delta \varepsilon_{k l}^{p}\right]-\frac{1}{\Delta t}\left[c_{0}+\alpha_{i j} \Delta \sigma_{i j}-\theta \alpha_{i j} C_{i j k l} \alpha_{k l}\right] \delta \theta+\Gamma_{i j}^{\varepsilon} \delta \varepsilon_{i j}+\Gamma^{\theta} \delta \theta=R_{i j}^{\varepsilon} \delta \varepsilon_{i j}+R^{\theta} \delta \theta,
$$

with

$$
R_{i j}^{\varepsilon}=-\frac{\theta}{\Delta t} \alpha_{k l} D_{k l i j}^{\varepsilon}+\Gamma_{i j}^{\varepsilon}, \quad R^{\theta}=-\frac{1}{\Delta t}\left[c_{0}+\alpha_{i j} \Delta \sigma_{i j}+\theta \alpha_{i j} D_{i j}^{\theta}\right]+\Gamma^{\theta}
$$

\section{$\mathrm{J}_{2}$ thermoelastoviscoplastic material with isotropic hardening}

Compared to the case of plasticity, viscoplasticity is characterized by the same set of equations except from the yield surface. A viscoplastic material is rather characterized by a family of equipotential surfaces, where the von Mises stress $\sigma^{\mathrm{vM}}$ is connected not only with the accumulated plastic strain $p$, but also with its rate $\dot{p}$. A typical viscoplastic law that arises as an extension of the previous case is characterized by the expression (Lemaitre and Chaboche, 2002)

$$
\dot{p}=\left\{\begin{array}{cc}
0, & \sigma^{\mathrm{vM}}-F-Y<0, \\
{\left[\frac{\sigma^{\mathrm{vM}}-F-Y}{K_{a}}\right]^{N_{a}},} & \sigma^{\mathrm{vM}}-F-Y \geq 0,
\end{array}\right.
$$

where $K_{a}$ and $N_{a}$ are material constants. The above expression permits to write a pseudo-yield surface of the form

$$
\Phi:=\Phi\left(\sigma_{i j}, F\right)=\sigma^{\mathrm{vM}}-F-Y-K_{a} \dot{p}^{1 / N_{a}} \leq 0, \quad \dot{p} \geq 0, \quad \Phi \dot{p}=0
$$

For this material, the thermomechanical tangent moduli are given from the expressions (A.12) and (A.16), where though

$$
\mathcal{A}=\Lambda_{i j} C_{i j k l} \Lambda_{k l}+\frac{\mathrm{d} F}{\mathrm{~d} p}+\frac{K_{a}}{N_{a} \Delta t} \dot{p}^{1 / N_{a}-1}
$$

Shape memory alloy (Lagoudas et al 2012)

For a model designed for shape memory alloys, the thermomechanical tangent moduli can be obtained in a similar fashion like the case ol elastoplasticity. Lagoudas et al. (2012) have presented such a constitutive law, based on a Gibbs free energy potential. The moduli $D_{i j k l}^{\varepsilon}$ and $D_{i j}^{\theta}$ are provided in Appendix A of Lagoudas et al. (2012), while the $R_{i j}^{\varepsilon}$ and $R^{\theta}$ can be computed from the equation (6.67) of the same manuscript. 


\section{References}

\section{References}

Aboudi, J., 2004. Micromechanics-based thermoviscoelastic constitutive equations for rubber-like matrix composites at finite strains. International Journal of Solids and Structures 41, 5611-5629.

Aboudi, J., Pindera, M.-J., Arnold, S. M., 2003. Higher-order theory for periodic multiphase materials with inelastic phases. International Journal of Plasticity 19 (6), 805-847.

Allaire, G., 1992. Homogenization and two-scale convergence. SIAM Journal on Mathematical Analysis 23, $1482-1518$.

Asada, T., Ohno, N., 2007. Fully implicit formulation of elastoplastic homogenization problem for two-scale analysis. International Journal of Solids and Structures 44 (22-23), 7261-7275.

Benaarbia, A., Chrysochoos, A., Robert, G., 2015. Thermomechanical behavior of PA6.6 composites subjected to low cycle fatigue. Composites Part B 76, 52-64.

Bensoussan, A., Lions, J., Papanicolaou, G., 1978. Asymptotic methods for periodic structures. North Holland, Amsterdam, The Netherlands.

Benveniste, Y., Dvorak, G. J., Chen, T., 1991. On diagonal and elastic symmetry of the approximate effective stiffness tensor oh heterogeneous media. Journal of the Mechanics and Physics of Solids 39 (7), 927-946.

Bravo-Castillero, J., Rodríguez-Ramos, R., Mechkour, H., Otero, J. A., Cabanas, J. H., Sixto, L. M., Guinovart-Díaz, R., Sabina, F. J., 2009. Homogenization and effective properties of periodic thermomagnetoelectroelastic composites. Journal of Mechanics of Materials and Structures 4 (5), 819-836.

Brenner, R., Suquet, P., 2013. Overall response of viscoelastic composites and polycrystals: exact asymptotic relations and approximate estimates. International Journal of Solids and Structures 50 (10), 1824-1838.

Cavalcante, M. A. A., Marques, S. P. C., Pindera, M. J., 2009. Transient thermomechanical analysis of a layered cylinder by the parametric finite-volume theory. Journal of Thermal Stresses 32, 112-134.

Cavalcante, M. A. A., Pindera, M. J., 2016. Generalized FVDAM theory for elasticplastic periodic materials. International Journal of Plasticity 77, 90-117.

Chaboche, J., Kanoute, P., Ross, A., 2005. On the capabilities of mean field approaches for the description of plasticity in metal matrix composites. International Journal of Plasticity 21, 1409-1434.

Charalambakis, N., 2010. Homogenization Techniques and Micromechanics. A Survey and Perspectives. Applied Mechanics Reviews 63 (3), 030803.

Chatzigeorgiou, G., Chemisky, Y., Meraghni, F., 2015. Computational micro to macro transitions for shape memory alloy composites using periodic homogenization. Smart Materials and Structures 24, 035009.

Chatzigeorgiou, G., Efendiev, Y., Charalambakis, N., Lagoudas, D. C., 2012. Effective Thermoelastic Properties of Composites with Periodicity in Cylindrical Coordinates. International Journal of Solids and Structures 49 (18), 2590-2603.

Desrumaux, F., Meraghni, F., Benzeggagh, M. L., 2001. Generalised Mori-Tanaka Scheme to Model Anisotropic Damage Using Numerical Eshelby Tensor. Journal of Composite Materials 35 (7), 603-624.

Ene, H. I., 1983. On linear thermoelasticity of composite materials. International Journal of Engineering Science 21 (5), $443-448$.

Fish, J., Shek, K., Pandheeradi, M., Shephard, M., 1997. Computational plasticity for composite structures based on mathematical homogenization: Theory and practice. Computer Methods in Applied Mechanics and Engineering 148, 53-73.

Germain, P., 1973. Cours de mécanique des milieux continus, Tome I: Théorie Générale. Masson, Paris, France.

Germain, P., 1982. Sur certaines définitions liées à l'énergie en mécanique des solides. International Journal of Engineering Science 20 (2), 245259

Germain, P., Nguyen, Q. S., Suquet, P., 1983. Continuum thermodynamics. Journal of Applied Mechanics 50, $1010-1020$.

Halphen, B., Nguyen, Q. S., 1975. Sur les matériaux standards généralisés. Journal de Mécanique 14 (1), 39-63. 
Hartl, D. J., Chatzigeorgiou, G., Lagoudas, D. C., 2010. Three-dimensional modeling and numerical analysis of rate-dependent irrecoverable deformation in shape memory alloys. International Journal of Plasticity 26 (10), 1485-1507.

Herzog, H., Jacquet, E., 2007. From a shape memory alloys model implementation to a composite behavior. Computational Materials Science 39, 365-375.

Hutchinson, J. W., 1976. Bounds and self-consistent estimates for creep of polycrystalline materials. Proceedings of Royal Society of London A $348,101-127$.

Javili, A., Chatzigeorgiou, G., Steinmann, P., 2013. Computational homogenization in magneto-mechanics. International Journal of Solids and Structures 50, 4197-4216.

Jendli, Z., Meraghni, F., Fitoussi, J., Baptist, D., 2009. Multi-scales modelling of dynamic behaviour for discontinuous fibre SMC composites. Composites Science and Technology 69 (1), 97-103.

Kalamkarov, A. L., Kolpakov, A. G., 1997. Analysis, design and optimization of composite structures. Wiley, West Sussex, UK.

Khatam, H., Pindera, M. J., 2010. Plasticity-triggered architectural effects in periodic multilayers with wavy microstructures. International Journal of Plasticity 26, 273-287.

Kruch, S., Chaboche, J. L., 2011. Multi-scale analysis in elasto-viscoplasticity coupled with damage. International Journal of Plasticity 27, 20262039.

Lagoudas, D., Hartl, D., Chemisky, Y., Machado, L., Popov, P., 2012. Constitutive model for the numerical analysis of phase transformation in polycrystalline shape memory alloys. International Journal of Plasticity 32-33, 155-183.

Lemaitre, J., Chaboche, J. L., 2002. Mechanics of solid materials. Cambridge University Press, Cambridge, UK.

Love, B., Batra, R., 2006. Determination of effective thermomechanical parameters of a mixture of two elastothermoviscoplastic constituents. International Journal of Plasticity 22, 1026-1061.

Maghous, S., Creus, G. J., 2003. Periodic homogenization in thermoviscoelasticity: case of multilayered media with ageing. International Journal of Solids and Structures 40, 851-870.

Meraghni, F., Desrumaux, F., Benzeggagh, M. L., 2002. Implementation of a constitutive micromechanical model for damage analysis in glass mat reinforced composite structures. Composites Science and Technology 62 (16), 2087-2097.

Mercier, S., Molinari, A., 2009. Homogenization of elastic-viscoplastic heterogeneous materials: Self-consistent and Mori-Tanaka schemes. International Journal of Plasticity 25, 1024-1048.

Mercier, S., Molinari, A., Berbenni, S., Berveiller, M., 2012. Comparison of different homogenization approaches for elasticviscoplastic materials. Modelling and Simulation in Materials Science and Engineering 20 (2), 024004.

Murat, F., Tartar, L., 1997. H-convergence, in Topics in the mathematical modelling of composite materials. In: Cherkaev, A., Kohn, R. (Eds.), Progress in Nonlinear Differential Equations and their Applications. Vol. 31. Birkhäuser, Boston, USA, pp. 21-43.

Pindera, M. J., Khatam, H., Drago, A. S., Bansal, Y., 2009. Micromechanics of spatially uniform heterogeneous media: A critical review and emerging approaches. Composites Part B: Engineering 40 (5), 349-378.

Ponte-Castañeda, P., 1991. The effective mechanical properties of nonlinear isotropic composites. Journal of the Mechanics and Physics of Solids $39,45-71$.

Qidwai, M. A., Lagoudas, D. C., 2000. Numerical Implementation of a Shape Memory Alloy Thermomechanical Constitutive Model Using Return Mapping Algorithms. International Journal for Numerical Methods in Engineering 47, 1123-1168.

Rosen, B. W., Hashin, Z., 1970. Effective thermal expansion coefficients and specific heats of composite materials. International Journal of Engineering Science $8,157-173$.

Sanchez-Palencia, E., 1978. Non-homogeneous media and vibration theory. In: Lecture Notes in Physics 127. Springer-Verlag, Berlin, Germany.

Sengupta, A., Papadopoulos, P., Taylor, R. L., 2012. A multiscale finite element method for modeling fully coupled thermomechanical problems in solids. International Journal for Numerical Methods in Engineering 91, 1386-1405.

Simo, J. C., Hughes, T. J. R., 1998. Computational Inelasticity. Springer-Verlag, New York, USA.

Sun, L. Z., Ju, J. W., 2004. Elastoplastic Modeling of Metal Matrix Composites Containing Randomly Located and Oriented Spheroidal Particles. 
Journal of Applied Mechanics 71, 774-785.

Suquet, P., 2012. Four exact relations for the effective relaxation function of linear viscoelastic composites. Comptes Rendus Mecanique 340, 387-399.

Suquet, P. M., 1987. Elements of homogenization for inelastic solid mechanics. In: Lecture Notes in Physics. Vol. 272. Springer, Berlin, Germany, pp. 193-278.

Temizer, İ., 2012. On the asymptotic expansion treatment of two-scale finite thermoelasticity. International Journal of Engineering Science 53, 74-84.

Terada, K., Kikuchi, N., 2001. A class of general algorithms for multi-scale analyses of heterogeneous media. Computer Methods in Applied Mechanics and Engineering 190, 5427-5464.

Tsalis, D., Baxevanis, T., Chatzigeorgiou, G., Charalambakis, N., 2013. Homogenization of elastoplastic composites with generalized periodicity in the microstructure. International Journal of Plasticity 51, 161-187.

Tsalis, D., Chatzigeorgiou, G., Tsakmakis, C., Charalambakis, N., 2015. Dissipation inequality-based periodic homogenization of wavy materials. Composites Part B 76, 89-104.

Tu, W., Pindera, M. J., 2014. Cohesive Zone-Based Damage Evolution in Periodic Materials Via Finite-Volume Homogenization. Journal of Applied Mechanics 81 (10), 101005.

Yu, Q., Fish, J., 2002. Multiscale asymptotic homogenization for multiphysics problems with multiple spatial and temporal scales: a coupled thermo-viscoelastic example problem. International Journal of Solids and Structures 39 (26), 6429-6452. 